\title{
Infinite-dimensional $p$-adic groups, semigroups of double cosets, and inner functions on Bruhat-Tits buildings
}

\author{
YuRY A. NERETIN 1
}

\begin{abstract}
We construct $p$-adic analogs of operator colligations and their characteristic functions. Consider a $p$-adic group $\mathbf{G}=\mathrm{GL}\left(\alpha+k \infty, \mathbb{Q}_{p}\right)$, its subgroup $L=\mathrm{O}\left(k \infty, \mathbb{Z}_{p}\right)$, and the subgroup $\mathbf{K}=\mathrm{O}\left(\infty, \mathbb{Z}_{p}\right)$ embedded to $L$ diagonally. We show that double cosets $\Gamma=\mathbf{K} \backslash \mathbf{G} / \mathbf{K}$ admit a structure of a semigroup, $\Gamma$ acts naturally in $\mathbf{K}$-fixed vectors of any unitary representations of $\mathbf{G}$. For each double coset we assign a 'characteristic function', which sends a certain Bruhat-Tits building to another building (buildings are finite-dimensional); image of the distinguished boundary is contained in the distinguished boundary. The latter building admits a structure of (Nazarov) semigroup, the product in $\Gamma$ corresponds to a point-wise product of characteristic functions.
\end{abstract}

\section{Degeneration of Iwahori-Hecke type algebras in the infinite dimensional limit}

1.1. Hypergroups of double cosets. Consider a group $G$ and its compact subgroup $K$. Consider double cosets $K \backslash G / K$, i.e., the quotient of $G$ with respect to the equivalence relation

$$
g \sim k_{1} g k_{2}, \quad \text { where } k_{1}, k_{2} \in K .
$$

Each double coset $\mathfrak{g}=K g K$ is equipped with a unique probability measure $\mu_{\mathfrak{g}}$, which is invariant with respect to left and right translations by elements of $K$. Convolution of measures $\mu_{\mathfrak{g}}, \mu_{\mathfrak{h}}$ can be represented in the form

$$
\mu_{\mathfrak{g}} * \mu_{\mathfrak{h}}=\int_{K \backslash G / K} \mu_{\mathfrak{r}}(\mathfrak{r}) d \sigma_{\mathfrak{g}, \mathfrak{h}}(\mathfrak{r}),
$$

where $\sigma_{\mathfrak{g}, \mathfrak{h}}$ is a positive probability measure on $K \backslash G / K$. Thus we get a map

$$
(\mathfrak{g}, \mathfrak{h}) \mapsto \sigma_{\mathfrak{g}, \mathfrak{h}}
$$

from $K \backslash G / K \times K \backslash G / K$ to the space of measures on $K \backslash G / K$. Such algebraic structures are called hypergroup $\}^{2}$. Also the map $g \mapsto g^{-1}$ induces an involution $\mu \mapsto \mu^{*}$ on the hypergroup,

$$
\left(\mu_{\mathfrak{g}} * \mu_{\mathfrak{h}}\right)^{*}=\mu_{\mathfrak{h}}^{*} * \mu_{\mathfrak{g}}^{*} .
$$

REMARK. We reformulate this in two forms.

\footnotetext{
${ }^{1}$ Supported by the grants FWF, P22122, and FWF, P25142.

${ }^{2}$ See, e.g., 1 .
} 
a) Denote by $\mathcal{M}(K \backslash G / K)$ the set of all (sign-indefinite) compactly supported measures on $G$, which are invariant with respect to left and right translations by elements of $K$. Then $\mathcal{M}(K \backslash G / K)$ is an algebra with respect to the convolution.

b) Let $G$ be a locally compact group with two-side invariant Haar measure $d g$. Consider the set $C(K \backslash G / K)$ of compactly supported left-right $K$-invariant continuous functions on $G$. Then $C(K \backslash G / K)$ is an algebra with respect to the convolution. Sometimes it is called (generalized) Iwahori-Hecke algebra.

Let $\rho$ be a unitary representation of $G$ in a Hilbert space $H$. Denote by $H^{K}$ the space of $K$-fixed vectors, by $P^{K}$ the projection operator to $H^{K}$. Let $g \in \mathfrak{g}$. Define the operator $H^{K} \rightarrow H^{K}$ given by

$$
\bar{\rho}(\mathfrak{g}):=\left.P^{K} \rho(g)\right|_{H^{K}} .
$$

It is easy to see that $\bar{\rho}(\mathfrak{g})$ depends on the double coset and not on a representative $g$. The operators $\bar{\rho}(g)$ also can be expressed as

$$
\bar{\rho}(g)=\left.\int_{K \times K} \rho\left(k_{1} g k_{1}\right) d k_{1} d k_{2}\right|_{H^{K}}=\left.\int_{K} \rho(k g) d k\right|_{H^{K}} .
$$

Also, we have a representation of the hypergroup in $H^{K}$ in the following sense:

$$
\bar{\rho}(\mathfrak{g} * \mathfrak{h})=\int \bar{\rho}(\mathfrak{r}) d \sigma_{\mathfrak{g}, \mathfrak{h}}(\mathfrak{r})
$$

Several special cases of this construction are widely used in representation theory, in particular for the following pairs $G \supset K$ :

$-G$ is a real semisimple Lie group and $K$ is the maximal compact subgroup; or $G$ is a compact Lie group and $K$ is a symmetric subgroup, [2], [3];

- $G$ is a finite Chevalley group, $K$ is a Borel subgroup, 4];

$-G$ is a $p$-adic semisimple group and $K$ is the Iwahori subgroup, [5.

Even for $(G, K)=(\mathrm{SL}(2, \mathbb{R}), \mathrm{SO}(2))$ the explicit expression for $\sigma_{\mathfrak{g}, \mathfrak{h}}$ is nontrivial, see [6].

For smaller subgroups $K \subset G$ in semisimple groups, the hypergroups $K \backslash$ $G / K$ became too complicated objects. For a noncompact subgroup $K$ there is no finite $K \times K$-invariant measure on $K \backslash G / K$. On the other hand, a convolution of infinite measures is not defined (except few exotic cases).

In 1970s R.S.Ismagilov and G.I.Olshanski observed that the situation can drastically change for infinite-dimensional groups. Now we discuss a real archetype of our $p$-adic construction.

1.2. Colligations. Denote by $\mathrm{U}(\infty)$ the group of all finitar $3^{3}$ infinite unitary matrices $g$. Denote by $\mathrm{O}(\infty) \subset \mathrm{U}(\infty)$ the group of real orthogonal

\footnotetext{
${ }^{3}$ An infinite matrix $g$ is finitary, if $g-1$ has only finite number of nonzero matrix elements
} 
matrices. We also use notation $\mathrm{U}(n+\infty)$ for the group of block finitary unitary matrices $\left(\begin{array}{ll}a & b \\ c & d\end{array}\right)$ of size $(n+\infty) \times(n+\infty)$. Consider double cosets

$$
K \backslash G / K=\mathrm{O}(\infty) \backslash \mathrm{U}(n+\infty) / \mathrm{O}(\infty),
$$

i.e., matrices $\left(\begin{array}{ll}a & b \\ c & d\end{array}\right) \in \mathrm{U}(n+\infty)$ determined up to the equivalence

$$
\left(\begin{array}{ll}
a & b \\
c & d
\end{array}\right) \sim\left(\begin{array}{ll}
1 & 0 \\
0 & u
\end{array}\right)\left(\begin{array}{ll}
a & b \\
c & d
\end{array}\right)\left(\begin{array}{ll}
1 & 0 \\
0 & v
\end{array}\right), \quad \text { where } u, v \in \mathrm{O}(\infty) .
$$

We call such equivalence classes by colligation 4 .

There is no Haar measure on $K$, therefore there are no natural measures on double cosets $K g K$, therefore we can not repeat the construction of a hypergroup $K \backslash G / K$.

However, there is a natural multiplication

$$
K \backslash G / K \times K \backslash G / K \rightarrow K \backslash G / K
$$

given by

$$
\left(\begin{array}{ll}
a & b \\
c & d
\end{array}\right) \circ\left(\begin{array}{ll}
p & q \\
r & t
\end{array}\right)=\left(\begin{array}{lll}
a & b & 0 \\
c & d & 0 \\
0 & 0 & 1
\end{array}\right)\left(\begin{array}{lll}
p & 0 & q \\
0 & 1 & 0 \\
r & 0 & t
\end{array}\right)=\left(\begin{array}{lll}
a p & b & c q \\
c p & d & c q \\
r & 0 & c q
\end{array}\right) .
$$

The matrix in the right-hand side has size $(n+\infty+\infty)$, we regard it as a matrix of size

$$
(n+(\infty+\infty)) \times(n+(\infty+\infty))=(n+\infty) \times(n+\infty) .
$$

Proposition 1.1 The o-multiplication is a well-defined associative operation on $K \backslash G / K$.

We also define an involution $\mathfrak{g} \mapsto \mathfrak{g}^{*}$ on $K \backslash G / K$ induced by the map $g \mapsto g^{*}$ (taking of adjoint operator). It is easy to verify the identity

$$
(\mathfrak{g} \circ \mathfrak{h})^{*}=\mathfrak{h}^{*} \circ \mathfrak{g}^{*} .
$$

Consider a unitary representation of $G=\mathrm{U}(n+\infty)$ in a Hilbert space $H$. As above consider the space $H^{K}$ of $K$-fixed vectors in $H$ and operators (1.1). The following multiplicativity theorem holds:

Theorem 1.2 (see [7, 8], Section IX.4) For any $\mathfrak{g}, \mathfrak{h} \in K \backslash G / K$,

$$
\bar{\rho}(\mathfrak{g}) \bar{\rho}(\mathfrak{h})=\bar{\rho}(\mathfrak{g} \circ \mathfrak{h}) .
$$

\footnotetext{
${ }^{4}$ This is a term from operator theory, a colligation (node) is the conjugacy class 1.10
} 
Also, for any $\mathfrak{g}$,

$$
\bar{\rho}\left(\mathfrak{g}^{*}\right)=\bar{\rho}(\mathfrak{g})^{*}
$$

These phenomena (semigroup structure on $K \backslash G / K$ and the multiplicativity) have no finite-dimensional analogs. However, for infinite-dimensional groups they are usual, see a discussion in Subsection 1.8 .

1.3. Characteristic functions. We wish to describe the o-multiplication on more usual language. For a matrix $g=\left(\begin{array}{ll}a & b \\ c & d\end{array}\right)$ we write the following equation

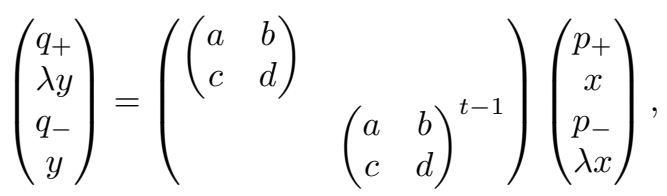

where $\lambda \in \mathbb{C}, x, y \in \ell_{2}, p_{ \pm}, q_{ \pm} \in \mathbb{C}^{n}$.

Eliminate variables $x, y$ from this system of equations, this is possible if

$$
\operatorname{det}\left(\lambda^{2} \bar{d}-d\right)
$$

is not identical zero. We get a dependence

$$
\left(\begin{array}{c}
q_{+} \\
q_{-}
\end{array}\right)=\chi_{g}(\lambda)\left(\begin{array}{c}
p_{+} \\
p_{-}
\end{array}\right)
$$

where $\lambda \mapsto \chi_{g}(\lambda)$ is a matrix-valued rational function on $\mathbb{C}$. It is called a characteristic function.

A characteristic function $\chi_{g}(\lambda)$ depends only on a double coset $\mathfrak{g}$ containing $g$ and not on $g$ itself.

Theorem 1.3 If $\chi_{\mathfrak{g}}(\lambda)$ and $\chi_{\mathfrak{h}}(\lambda)$ are well-defined, then

$$
\chi_{\mathfrak{g} \circ \mathfrak{h}}(\lambda)=\chi_{\mathfrak{g}}(\lambda) \chi_{\mathfrak{h}}(\lambda) .
$$

Also,

$$
\chi_{\mathfrak{g}^{*}}(\lambda)=\chi_{\mathfrak{g}^{*}}\left(\lambda^{-1}\right)^{-1}
$$

1.4. Reformulation. The language of Grassmannians. Fix $\lambda$. Consider the set $\mathcal{X}_{\mathfrak{g}}(\lambda)$ of all $\left(q_{+}, q_{-} ; p_{+}, p_{-}\right) \in \mathbb{C}^{2 n} \oplus \mathbb{C}^{2 n}$ such that there are $x, y$ satisfying (1.3). Evidently, $\mathcal{X}_{\mathfrak{g}}(\lambda)$ is a linear subspace. Notice, that at a nonsingular point of the function $\chi_{\mathfrak{g}}(\lambda)$, the subspace $\mathcal{X}_{\mathfrak{g}}(\lambda)$ is the graph of the operator $\chi_{\mathfrak{g}}(\lambda): \mathbb{C}^{n} \rightarrow \mathbb{C}^{n}$.

Next, we extend the function $\mathcal{X}_{\mathfrak{g}}(\lambda)$ to the Riemann sphere $\overline{\mathbb{C}}=\mathbb{C} \cup \infty$ in the following way. We write the equation

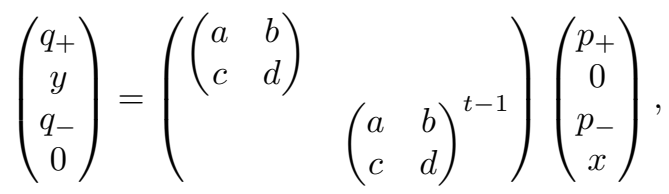

and consider the set $\mathcal{X}_{\mathfrak{g}}(\infty)$ of all $\left(q_{+}, q_{-} ; p_{+}, p_{-}\right) \in \mathbb{C}^{2 n} \oplus \mathbb{C}^{2 n}$ such that the equation (1.5) has a solution. 
Theorem 1.4 a) $\operatorname{dim} \mathcal{X}_{\mathfrak{g}}(\lambda)=2 n$ for all $\lambda \in \overline{\mathbb{C}}$.

b) For any $\mathfrak{g}$ the map $\lambda \mapsto \mathcal{X}_{\mathfrak{g}}(\lambda)$ is holomorphic on $\overline{\mathbb{C}}$.

Emphasize that the characteristic function $\mathcal{X}_{\mathfrak{g}}(\lambda)$ is well-defined for all double cosets $\mathfrak{g}$.

Next, we explain how to interpret formula (1.4) on the language of Grassmannian.

Let $V, W$ be linear spaces. We say that a linear relation $L: V \rightrightarrows W$ is a subspace $L \subset V \oplus W$.

ExAmPle. Let $A: V \rightarrow W$ be a linear operator. Then its graph graph $(A) \subset$ $V \oplus W$ is a linear relation. The set of all linear subspaces in $V \oplus W$ consists of $\operatorname{dim} V+\operatorname{dim} W$ components. Graphs of operators constitute an open dense subspace in one of components.

Consider two linear relations $L: V \rightrightarrows W, M: W \rightrightarrows Y$. Define their product $L M: V \rightrightarrows Y$ as the set of $(r, p) \in V \oplus Y$ such that there exists $q \in W$ such that $(r, q) \in L,(q, p) \in M$.

Also, for a linear relation $L: V \rightrightarrows W$ we define the kernel $\operatorname{ker} L \subset V$ and the indefinity indef $L \subset W$,

$$
\operatorname{ker} L:=L \cap(V \oplus 0), \quad \text { indef } L:=L \cap(0 \oplus W) .
$$

Theorem 1.5 For any $\mathfrak{g}, \mathfrak{h}$ and each $\lambda \in \overline{\mathbb{C}}$,

$$
\mathcal{X}_{\mathfrak{g} \circ \mathfrak{h}}(\lambda)=\mathcal{X}_{\mathfrak{g}}(\lambda) \mathcal{X}_{\mathfrak{h}}(\lambda) \text {. }
$$

1.5. Conditions for characteristic functions. We equip the space $\mathbb{C}^{n} \oplus$ $\mathbb{C}^{n}$ with a standard skew-symmetric bilinear form determined by the matrix $\left(\begin{array}{cc}0 & 1 \\ -1 & 0\end{array}\right)$. We regard vectors $\left(p_{+}, p_{-}\right)$and $\left(q_{+}, q_{-}\right)$as elements of $\mathbb{C}^{n} \oplus \mathbb{C}^{n}$. Denote by $\operatorname{Sp}(2 n, \mathbb{C})$ the group of operators preserving this form.

Equip the space $\left(\mathbb{C}^{n} \oplus \mathbb{C}^{n}\right) \oplus\left(\mathbb{C}^{n} \oplus \mathbb{C}^{n}\right)$ by the difference of skew-symmetric forms, i.e. by the form with matrix

$$
\left(\begin{array}{cccc}
0 & 1 & 0 & 0 \\
-1 & 0 & 0 & 0 \\
0 & 0 & 0 & -1 \\
0 & 0 & 1 & 0
\end{array}\right)
$$

We regard vectors $\left(p_{+}, p_{-}, q_{+}, q_{-}\right)$as elements of this space.

Proposition 1.6 (see [8], IX.4)

a) Outside poles, values of $\chi_{\mathfrak{g}}(\lambda)$ are contained in the complex symplectic group $\operatorname{Sp}(2 n, \mathbb{C})$.

b) The characteristic function $\mathcal{X}_{\mathfrak{g}}(\lambda)$ takes values in the Lagrangian Grassmannian5.

\footnotetext{
${ }^{5}$ Recall that a subspace $L$ in a $2 m$-dimensional linear space equipped with a nondegenerate skew-symmetric bilinear form is Lagrangian if the form vanishes on $L$ and $\operatorname{dim} L=m$, see, e.g., 9], Section 3.1.
} 
Second, consider the Hermitian form $M$ on $\mathbb{C}^{n} \oplus \mathbb{C}^{n}$ determined by the matrix $\left(\begin{array}{cc}0 & i \\ -i & 0\end{array}\right)$. Denote by $\mathrm{U}(n, n)$ the group of matrices preserving $M$.

We say that a linear operator $A$ in $\mathbb{C}^{n} \oplus \mathbb{C}^{n}$ is an $M$-contraction (see, e.g., [9], Section 2.7), if for all vectors $v$ we have

$$
M(A v, A v) \leqslant M(v, v) .
$$

We say that $A$ is an $M$-dilatation if $M(A v, A v) \geqslant M(v, v)$.

Also, equip the space $\left(\mathbb{C}^{n} \oplus \mathbb{C}^{n}\right) \oplus\left(\mathbb{C}^{n} \oplus \mathbb{C}^{n}\right)$ with the difference of Hermitian forms, i.e. with a form $\widetilde{M}$ given by

$$
\left(\begin{array}{cccc}
0 & i & 0 & 0 \\
-i & 0 & 0 & 0 \\
0 & 0 & 0 & -i \\
0 & 0 & i & 0
\end{array}\right)
$$

Proposition 1.7 (see [8], Section IX.4) Let $\chi_{\mathfrak{g}}(\lambda)$ be well-defined. Then:

a) If $|\lambda|=1$, then $\chi_{\mathfrak{g}}(\lambda) \in \mathrm{U}(n, n)$.

b) If $|\lambda|<1$, then $\chi_{\mathfrak{g}}(\lambda)$ is an $M$-contraction.

c) If $|\lambda|>1$, then $\chi_{\mathfrak{g}}(\lambda)$ is an $M$-dilatation.

Proposition 1.8 (see [8], Section IX.4)

a) If $|\lambda|=1$, then the subspace $\mathcal{X}_{\mathfrak{g}}(\lambda)$ is $\widetilde{M}$-isotropic.

b) If $|\lambda|<1$, then the form $\widetilde{M}$ is positive semi-definite on the subspace $\mathcal{X}_{\mathfrak{g}}(\lambda)$.

c) If $|\lambda|>1$, then the form $\widetilde{M}$ is negative semi-definite on the subspace $\mathcal{X}_{\mathfrak{g}}(\lambda)$.

d) If $|\lambda|<1$, then the from $M$ is strictly positive definite on 6 ker $\mathcal{X}_{\mathfrak{g}}(\lambda)$. Also $M$ is negative definite on indef $\mathcal{X}_{\mathfrak{g}}(\lambda)$.

Characteristic functions also satisfy to the following condition of symmetry at 0

$$
\chi_{\mathfrak{g}}(-\lambda)=\left(\begin{array}{cc}
1 & 0 \\
0 & -1
\end{array}\right) \chi_{\mathfrak{g}}(\lambda)\left(\begin{array}{cc}
1 & 0 \\
0 & -1
\end{array}\right)^{-1} .
$$

On the language of Grassmannians this means

$$
\left(p_{+}, p_{-}, q_{+}, q_{-}\right) \in \mathcal{X}(\lambda) \Leftrightarrow\left(p_{+},-p_{-}, q_{+},-q_{-}\right) \in \mathcal{X}(\lambda) .
$$

Theorem 1.9 Any holomorphic map $\mathcal{X}$ from $\overline{\mathbb{C}}$ to the Lagrangian Grassmannian satisfying the conditions of Proposition 1.8 and condition (1.7) is a characteristic function of a double coset $\mathfrak{g}$.

\footnotetext{
${ }^{6}$ This condition contains additional information only at points $\lambda$, where $\mathcal{X}(\lambda)$ is not a graph of an operator. By statement b) $M$ is positive semi-definite on the kernel.
} 
1.6. Central extension. A characteristic function is not sufficient for a reconstruction of a double coset, in fact matrices of the form

$$
\left(\begin{array}{lll}
a & b & 0 \\
c & d & 0 \\
0 & 0 & e
\end{array}\right) \begin{aligned}
& \} n \\
& \} \infty \\
& \} \infty
\end{aligned}
$$

with fixed $a, b, c, d$ and arbitrary $e$ have the same characteristic function. Let us introduce an additional invariant. We write the equation

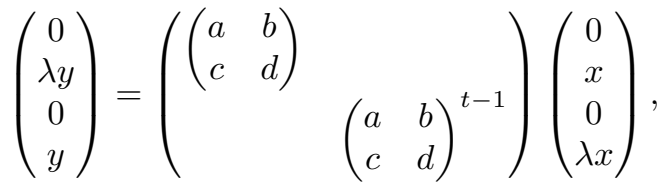

as an equation for $x, y$. Denote by $n_{\mathfrak{g}}(\lambda)$ the dimension of the space of solutions of this equation. Then

$-n_{\mathfrak{g}}(\lambda)=0$ for all but a finite number of values of $\lambda$;

$-n_{\mathfrak{g}}(\lambda)=0$ if $|\lambda| \neq 1$

$-n_{\mathfrak{g}}(\lambda)=n_{\mathfrak{g}}(-\lambda)$

$-n_{\mathfrak{g}}( \pm 1)=\infty$.

Thus we get a finite set with multiplicities (we call it divisor).

Theorem 1.10 7 A double coset is uniquely determined by its characteristic function $\mathcal{X}$ and the divisor $n$.

Theorem 1.11 [8] IX.4.5)

$$
n_{\mathfrak{g} \circ \mathfrak{h}}(\lambda)=n_{\mathfrak{g}}(\lambda)+n_{\mathfrak{g}}(\mathfrak{h} ; \lambda)+\operatorname{dim}\left(\operatorname{indef} \mathcal{X}_{\mathfrak{h}}(\lambda) \cap \operatorname{ker} \mathcal{X}_{\mathfrak{g}}(\lambda)\right) .
$$

Double cosets corresponding matrices

$$
\left(\begin{array}{lll}
1 & 0 & 0 \\
0 & 1 & 0 \\
0 & 0 & e
\end{array}\right) \begin{aligned}
& \} n \\
& \{\infty \\
& \} \infty
\end{aligned}
$$

is the center of the semigroup $K \backslash G / K$. The quotient of $K \backslash G / K$ with respect to the center is isomorphic to the semigroup of rational matrix-values functions described above.

\footnotetext{
${ }^{7}$ This and previous statements are given in 8], .IX.4.8 without formal proof. In fact, a proof is contained in the same book, Addendum E. Precisely, in Subsection E.4 it is shown how to reduce our statements to the standard theorem (see 10]) 'pure unitary operator node is determined by its characteristic function'. In fact, we only need this theorem for finitary matrices and rational characteristic functions.
} 
1.7. Degeneration of hypergroups of double cosets. Let $N>k$. Embed $\mathrm{U}(n+k)$ to $\mathrm{U}(n+k+N)$ by

$$
\iota_{N}:\left(\begin{array}{cc}
A & B \\
C & D
\end{array}\right) \mapsto\left(\begin{array}{ccc}
A & B & 0 \\
C & D & 0 \\
0 & 0 & 1
\end{array}\right) .
$$

Embed $\mathrm{U}(k+N)$ to $\mathrm{U}(n+k+N)$ by

$$
\left(\begin{array}{cc}
\alpha & \beta \\
\gamma & \delta
\end{array}\right) \mapsto\left(\begin{array}{ccc}
1 & 0 & 0 \\
0 & \alpha & \beta \\
0 & \gamma & \delta
\end{array}\right) .
$$

Fix matrices $g=\left(\begin{array}{ll}q & b \\ c & d\end{array}\right), h=\left(\begin{array}{ll}p & q \\ r & t\end{array}\right) \in \mathrm{U}(n+k)$. Then for $N>k$ a matrix $\iota_{N}(g) \circ \iota_{N}(h)$ is well-defined as an element of $\mathrm{U}(k+N) \backslash \mathrm{U}(n+k+N) / \mathrm{U}(k+N)$.

We equip the group $\mathrm{U}(n+k+N)$ with the metric induced by the operator norm in Euclidean $\mathbb{C}^{n+k+N}$.

Proposition 1.12 Fix $g, h \in \mathrm{U}(n+k)$ as above. Consider the corresponding double cosets

$$
\mathfrak{g}_{N}, \mathfrak{h}_{N} \in \mathrm{U}(k+N) \backslash \mathrm{U}(n+k+N) / \mathrm{U}(k+N)
$$

and the measure

$$
\varkappa_{N}=\mu_{\mathfrak{g}_{N}} * \mu_{\mathfrak{h}_{N}}
$$

Then for each $\varepsilon>0, \delta>0$ there exists $N$ such that the measure $\varkappa_{N}$ of $\varepsilon$ neighborhood of $\iota_{N}(g) \circ \iota_{N}(h)$ is $>1-\delta$.

See [7, 11], [12.

1.8. Semigroups of double cosets. The first example of multiplication of double cosets was discovered by Ismagilov [13, he considered the group $G=\mathrm{SL}(2, k)$ over a non-Archimedian normed non locally compact field $k$. The subgroup $K$ is the group $\mathrm{SL}(2, o)$ over integer elements of $k$. The double cosets are parametrized by non-negative integers $\mathbb{Z}_{+}$, and the operation $\circ$ is the usual addition. The multiplicativity theorem allows to classify spherical functions (see also [14). Olshanski [15] showed that this semigroup is a limit of hypergroups $\mathrm{SL}\left(2, \mathbb{Z}_{p}\right) \backslash \mathrm{SL}\left(2, \mathbb{Q}_{p}\right) / \mathrm{SL}\left(2, \mathbb{Z}_{p}\right)$ as $p \rightarrow \infty$.

Next, consider a series of Riemannian symmetric spaces $G(n) / K(n)$ (an example is $\mathrm{U}(n) / \mathrm{O}(n))$. Olshanski 7], 11] showed that the same phenomena hold for any pair $G(k+\infty) \supset K(\infty)$. Also he described such semigroups for infinite symmetric groups. As far as we know description of such objects, they became a tool of the representation theory. On the other hand, it seems that such structure are interesting by themselves.

In $[8$, Section 8.5, the author observed that multiplications on $K \backslash G / K$ are quite usual for infinite-dimensional groups (see also [16, [17]). In fact this happened more-or-less always if $K$ is one of the following groups: 
1) $K$ is a complete infinite unitary group, orthogonal group, or symplectic (quaterninic unitary) group (or a product of several copies of such groups);

2) $K$ is the infinite symmetric group $S(\infty)$;

$3) K$ is the group of automorphisms of a measure space;

These groups are infinite-dimensional imitation of compact groups (but they are neither compact, nor locally compact) apparently some other examples also exist (for instance, below we discuss $K=\mathrm{O}\left(\infty, \mathbb{Z}_{p}\right)$ ).

For precise general theorems, see [16, 17. To explore them we need explicit descriptions of $K \backslash G / K$, such descriptions recently were obtained in [18, 20], [16, [17.

1.9. Inner functions. Recall a definition of inner functions.

1) A holomorphic function $f(z)$ in a unit disk $|z|<1$ is called inner, if $|f(z)|<1$ for $|z|<1$ and

$$
\lim _{r \rightarrow 1^{-}}\left|f\left(r e^{i \theta}\right)\right|=1 \quad \text { a.s. } \theta \in[0,2 \pi],
$$

where $z=r e^{i \theta}$ and $r, \theta$ are rea 8 . On this topic, see, e.g., 21. It can be shown that limit (1.8) can be replaced a.s. by the nontangential limit

$$
\lim _{z \rightarrow e^{i \theta},\left|\arg \frac{e^{i \theta}-z}{e^{i \theta}}\right| \leqslant \pi / 2-\varepsilon} f(z),
$$

where $\varepsilon>0$ is fixed (in fact we consider a limit over the angle whose vertex is $e^{i \theta}$, the bisector is $t e^{i \theta}$, and the value of the angle is $\pi-2 \varepsilon$.

2) A homomorphic matrix-valued (operator-valued) function $f(z)$ in the unit disk is called inner if $\|f(z)\| \leqslant 1$ for $|z|<1$ and boundary values of $f$ on the circle are unitary (see Livshits [22, Potapov 23]). Consider an operator $d$ closed to unitary (one of possible variants $\operatorname{rk}\left(d d^{*}-1\right)=\operatorname{rk}\left(d^{*} d-1\right)<\infty$ ) with $\|d\|=1$. We are interested its properties up to conjugations $d \mapsto u d u^{-1}$, where $u$ is unitary. Build a larger unitary matrix $g=\left(\begin{array}{ll}a & b \\ c & d\end{array}\right)$ including $d$ as a block. We consider $g$ up to the equivalence

$$
\left(\begin{array}{ll}
a & b \\
c & d
\end{array}\right) \sim\left(\begin{array}{ll}
1 & 0 \\
0 & u
\end{array}\right)\left(\begin{array}{ll}
a & b \\
c & d
\end{array}\right)\left(\begin{array}{cc}
1 & 0 \\
0 & u^{-1}
\end{array}\right), \quad \text { where } u \in \mathrm{U}(\infty)
$$

Assign to $g$ the expression (characteristic function) by

$$
\chi(\lambda)=a+\lambda b(1-\lambda d)^{-1} c .
$$

Such functions (under some conditions on $d$ ) are inner functions $\theta(z)$ in the unit disk. Invariant subspaces of $d$ are in one-to-one correspondence with divisors of $\theta$ in the class of inner functions. The product of inner functions corresponds to the product of conjugacy classes (1.10) by formula (1.2).

\footnotetext{
${ }^{8}$ We can not write a limit as $z \rightarrow e^{i \theta}$, an inner function can be discontinuous at all points of the circle.
} 
3) More generally, consider a pseudo-Euclidean space. We say that a meromorphic matrix-valued function $f$ in the disk is inner if it is indefinite contractive in the disk and pseudo-unitary on the unit circle. Such functions arise in the same context but the condition $\|d\| \leqslant 1$ is omitted.

The characteristic function of double cosets defined above are inner in this sense.

4) Denote by $B_{n}$ the set of all $n \times n$ complex symmetric matrix with norm $<1 ; B_{n}$ also is an Hermitian symmetric space

$$
B_{n}=\mathrm{U}(n, n) / \mathrm{U}(n) \times \mathrm{U}(n),
$$

its distinguished boundary (Shilov boundary) consist of unitary matrices.

In 20, 16] there were considered various semigroups of double cosets on infinite-dimensional classical groups. For instance, consider group $G=\mathrm{U}(\alpha+$ $k \infty)$ consisting of block unitary matrices of size $\alpha+\infty+\cdots+\infty$. Consider its subgroup $L=\mathrm{U}(\infty)$ embedded to $G$ in the block diagonal way. Consider the subgroup $K=\mathrm{O}(\infty) \subset L$ embedded to $\mathrm{U}(\infty)$ in the natural way. Then $K \backslash G / K$ is a semigroup. Characteristic functions 20] are inner functions in $B_{k} \times B_{k}$ taking values at the space of $2 \alpha \times 2 \alpha$-matrices. This means that values of a function are $M$-contractions inside $B_{k} \times B_{k}$ and are pseudounitary on the Shilov boundary $\mathrm{U}(n) \times \mathrm{U}(n)$. The product of double cosets corresponds to the product of characteristic functions.

It is possible to vary the definition and to regard a characteristic function as a map $B_{k} \times B_{k} \rightarrow B_{2 \alpha}$.

1.10. Infinite-dimensional $p$-adic groups. Representation theory of infinite-dimensional classical groups (see, e.g., [24, [25, [7, [8, 26], 27, [16]) and infinite symmetric groups (see, e.g., [28, [29, [18]) exists and is welldeveloped. There were several recent works concerning infinite-dimensional classical groups over finite fields (see [30, 31, 32]).

Few is known about infinite-dimensional $p$-adic groups. There are the following works:

1) Work of Nazarov [33, 34 on the Weil representation of an infinitedimensional group $\operatorname{Sp}\left(2 \infty, \mathbb{Q}_{p}\right)$. Existence of such representation is more-or-less evident. However, the Weil representation of $\operatorname{Sp}(2 n, \mathbb{R})$ and $\operatorname{Sp}(2 \infty, \mathbb{R})$ admits a continuation to a certain complex domain $\Gamma$ (if $n<\infty$, then $\Gamma$ is a semigroup parametrized by complex symmetric $2 n \times 2 n$ matrices with norm $<1$, see, e.g., 8], Section 4.2, 9], Section 5.1). Nazarov constructed an analog of $\Gamma$ for $p$-adic case, see below Section 3 (for more details, see [9], Sections 10.7, 11.2)

2) A construction of Hua measures on $p$-adic Grassmannians and on the inverse limit of $p$-adic Grassmannians in 35. This is an analog of inverse limits of compact symmetric spaces (see [36]) and of symmetric groups (see [29]). Recall that in latter two cases there exists a substantial harmonic analysis on such inverse limits, see [27, [29].

3) The group of diffeomorphisms of $p$-adic projective line is an object similar to the group of diffeomorphisms of the circle (many constructions of representations of the latter group survive in $p$-adic case, [37]). 
1.11. A $p$-adic example. Here we briefly discuss a $p$-adic object, which is related to the topic of this paper but more simple. Let $\mathbb{Q}_{p}$ be a $p$-adic field, $\mathbb{Z}_{p} \subset$ $\mathbb{Q}_{p}$ be the ring of $p$-adic integers. Denote by $\operatorname{GL}\left(\infty, \mathbb{Q}_{p}\right)$ the group of finitary invertible matrices over $\mathbb{Q}_{p}$. Consider conjugacy classes of $\operatorname{GL}\left(\alpha+\infty, \mathbb{Q}_{p}\right)$ with respect to the subgroup $\mathrm{GL}\left(\infty, \mathbb{Z}_{p}\right)$,

$$
\left(\begin{array}{ll}
a & b \\
c & d
\end{array}\right) \sim\left(\begin{array}{ll}
1 & 0 \\
0 & u
\end{array}\right)\left(\begin{array}{ll}
a & b \\
c & d
\end{array}\right)\left(\begin{array}{cc}
1 & 0 \\
0 & u^{-1}
\end{array}\right), \quad \text { where } u \in \operatorname{GL}\left(\infty, \mathbb{Z}_{p}\right) .
$$

Such conjugacy classes admit a natural o-multiplication by formula (1.2), this multiplication is a well-defined associative operation on the space of conjugacy classes. We wish to construct an analog of characteristic functions.

First, choose a sufficiently large $m$ such that a matrix $\left(\begin{array}{ll}a & b \\ c & d\end{array}\right)$ is actually contained in $\operatorname{GL}\left(\alpha+m, \mathbb{Q}_{p}\right)$. Consider a lattic $9 \Omega \mathbb{Q}_{p}^{2}$. For this lattice we consider the lattice

$$
R \otimes \mathbb{Z}_{p}^{m} \subset \mathbb{Q}_{p}^{2} \otimes \mathbb{Q}_{p}^{m} \simeq \mathbb{Q}_{p}^{m} \oplus \mathbb{Q}_{p}^{m}
$$

We write an equation

$$
\left(\begin{array}{l}
v \\
y
\end{array}\right)=\left(\begin{array}{ll}
a & b \\
c & d
\end{array}\right)\left(\begin{array}{l}
u \\
x
\end{array}\right) .
$$

Next, consider the set $\chi(R)$ of all pairs $(v, u) \in \mathbb{Q}_{p}^{\alpha} \oplus \mathbb{Q}_{p}^{\alpha}$ for which there exists $y \oplus x \in R \otimes \mathbb{Z}_{p}^{m}$ such that the equality (1.12) is satisfied. Then $\chi(R)$ is a $\mathbb{Z}_{p^{-}}$ submodule in $\mathbb{Q}_{p}^{\alpha} \oplus \mathbb{Q}_{p}^{\alpha}$, which can be regarded as a relation $\mathbb{Q}_{p}^{\alpha} \rightrightarrows \mathbb{Q}_{p}^{\alpha}$. The o-product corresponds to point-wise product of functions $\chi(R)$ with values in relations.

We also point out that these functions are compatible with the structure of Bruhat-Tits buildings and are inner in a reasonable sense. Both phenomena are discussed below for more sophisticated objects.

1.12. Purpose of the paper. We wish to describe multiplication of double cosets on $p$-adic groups and to obtain analogs of characteristic functions. For a double coset we assign a simplicial map from a Bruhat-Tits building $\Omega$ to a Bruhat-Tits building $\Xi$ such that the image of the distinguished boundary is contained in the distinguished boundary. We also have a structure of a semigroup on the set of vertices of the building $\Xi$ (the Nazarov semigroup) and the product of double cosets corresponds to pointwise product of functions $\Omega \rightarrow \Xi$.

Our construction is not a final solution of the problem 10

1.13. A non-properly understood link. In fact our main construction below is organized as an extension of rational maps of $p$-adic Grassmannians to simplicial maps of Bruhat-Tits buildings. Also, our construction admits an

\footnotetext{
${ }^{9}$ see a definition in Subsection 3.1

${ }^{10}$ First, we do not introduce an analog of the 'divisor'. Secondly, [19] suggests that complete data separating double cosets must contain a sequence of characteristic functions determined on the increasing sequence of buildings. A construction of complete data in a real case in [19] is based on classical invariant theory, which does not valid over the ring $\mathbb{Z}_{p}$.
} 
automatic pass to algebraic extensions. Constructions of such type are investigated in theory of Berkovich analytic spaces, see, e.g., [39, 40]. However their extensions are rigid, and our extensions depend on additional data11. So I can not understand relations of our constructions and Berkovich theory.

1.14. Notation. Let

- $A^{t}$ be the transposed matrix;

$-1_{\alpha}, 1_{V}$ be the unit matrix of order $\alpha$, the unit operator in a space $V$;

- $\mathbb{Q}_{p}$ be the $p$-adic field;

$-\mathbb{Z}_{p}$ be the ring of $p$-adic integers;

- $\mathbb{Q}_{p}^{\times}, \mathbb{C}^{\times}$be multiplicative groups of $\mathbb{Q}_{p}, \mathbb{C}$.

We denote the standard character $\mathbb{Q}_{p} \rightarrow \mathbb{C}^{\times}$by $\exp \{2 \pi i a\}$. For $a=$ $\sum_{\geqslant-N} a_{j} p^{j}$, where $a_{j}=0,1, \ldots, p-1$, we set

$$
\exp \{2 \pi i a\}=\exp \left\{2 \pi i \sum_{j \geqslant-N} a_{j} p^{j}\right\}:=\exp \left\{2 \pi i \sum_{j:-1 \geqslant j \geqslant-N} a_{j} p^{j}\right\}
$$

Below we define:

- the groups $\mathrm{GL}\left(n, \mathbb{Q}_{p}\right), \operatorname{Sp}\left(2 n, \mathbb{Q}_{p}\right), \operatorname{Sp}\left(2 n, \mathbb{Q}_{p}\right), \mathrm{O}\left(n, \mathbb{Z}_{p}\right), \operatorname{GL}\left(\infty, \mathbb{Z}_{p}\right)$, $\operatorname{Sp}\left(2 \infty, \mathbb{Q}_{p}\right)$, etc., Subsection 2.1

$-V_{ \pm}$, formula (2.1);

- groups $\mathbf{G}=\mathrm{GL}\left(\alpha+k \infty, \mathbb{Q}_{p}\right), \mathbf{K}=\mathrm{O}\left(\infty, \mathbb{Z}_{p}\right)$, Subsection 2.2

$-\mathfrak{g} \star \mathfrak{h}$, the product of double cosets, Subsection 2.2,

- $\mathfrak{g}^{*}$, the involution on double cosets, Subsection 2.5.

$-R_{\downarrow}, R^{\uparrow}$, , Subsection 3.1

$-R_{j} \nearrow R$, rigid convergence, 3.4. tion 3.5 .

- $\operatorname{LMod}(V)$, LLat $(V)$, LGr $(V)$, spaces of Lagrangian submodules, Subsec-

- $\Delta(V), \operatorname{Bd}(V)$, buildings, Subsections 3.6, 3.8,

$-P: V \rightrightarrows W, \operatorname{ker} P$, indef $P, \operatorname{dom} P, \operatorname{im} P, P^{\square}$, Subsection 3.9.

— Naz, Naz, Naz, the Nazarov category, Subsections 3.12, 3.14

- We, the Weil representation, Subsection 3.16

$-\chi_{\mathfrak{g}}(Q, T)$, a characteristic function, Subsection 4.1 .

\footnotetext{
${ }^{11}$ Below rational maps of Grassmannians originate from double cosets$$
\mathrm{O}\left(\infty, \mathbb{Q}_{p}\right) \backslash \mathrm{GL}\left(\alpha+k \infty, \mathbb{Q}_{p}\right) / \mathrm{O}\left(\infty, \mathbb{Q}_{p}\right)
$$

(see Proposition 4.11) maps of Bruhat-Tits buildings from double cosets

$$
\mathrm{O}\left(\infty, \mathbb{Z}_{p}\right) \backslash \mathrm{GL}\left(\alpha+k \infty, \mathbb{Q}_{p}\right) / \mathrm{O}\left(\infty, \mathbb{Z}_{p}\right)
$$

Therefore we get many maps of Bruhat-Tits buildings with the same restriction to a distinguished boundary, i.e., to the Grassmannian.
} 


\section{Multiplication of double cosets}

2.1. Groups. By $V=\mathbb{Q}_{p}^{n}$ we denote linear spaces over $\mathbb{Q}_{p}$. Denote by $\operatorname{GL}\left(n, \mathbb{Q}_{p}\right)=\mathrm{GL}(V)$ the group of invertible linear operators in $\mathbb{Q}_{p}^{n}$; by $\mathrm{GL}\left(n, \mathbb{Z}_{p}\right)$ the group of all matrices $g$ with integer elements, such that $g^{-1}$ have integer elements.

Consider a space $V=\mathbb{Q}_{p}^{2 n}$ equipped with a non-degenerate skew-symmetric bilinear form $B_{V}$, say $\left(\begin{array}{cc}0 & 1 \\ -1 & 0\end{array}\right)$. The symplectic group $\operatorname{Sp}\left(2 n, \mathbb{Q}_{p}\right)$ is the group of matrices preserving this form, $\operatorname{Sp}\left(2 n, \mathbb{Z}_{p}\right)$ is the group of symplectic matrices with integer elements. We also denote

$$
V_{+}:=\mathbb{Q}_{p}^{n} \oplus 0, \quad V_{-}=0 \oplus \mathbb{Q}_{p}^{n} .
$$

Also, consider a space $\mathbb{Q}_{p}^{n}$ equipped with the standard symmetric bilinear form $(v, w)=\sum v_{j} w_{j}$. We denote by $\mathrm{O}\left(n, \mathbb{Q}_{p}\right)$ the group of all matrices preserving this form 12 .

By $\operatorname{GL}\left(\infty, \mathbb{Q}_{p}\right)$ we denote the group of all infinite invertible matrices over $\mathbb{Q}_{p}$ such that $g-1$ has only finite number of non-zero elements. We call such matrices finitary. We define $\mathrm{GL}\left(\infty, \mathbb{Z}_{p}\right), \operatorname{Sp}\left(2 \infty, \mathbb{Q}_{p}\right), \operatorname{Sp}\left(2 \infty, \mathbb{Z}_{p}\right), \mathrm{O}\left(\infty, \mathbb{Z}_{p}\right)$ in the same way.

\subsection{Multiplication of double cosets. Let}

$$
\mathbf{G}:=\mathrm{GL}\left(\infty, \mathbb{Q}_{p}\right):=\mathrm{GL}\left(\alpha+k \infty, \mathbb{Q}_{p}\right)
$$

be the group of finitary block $(\alpha+\infty+\cdots+\infty) \times(\alpha+\infty+\cdots+\infty)$ - matrices (there are $k$ copies of $\infty$ ). By $\mathbf{K}$ we denote the group

$$
\mathbf{K}=\mathrm{O}\left(\infty, \mathbb{Z}_{p}\right)
$$

embedded to $\mathbf{G}$ by the rule

$$
\mathfrak{I}: u \mapsto\left(\begin{array}{cccc}
1_{\alpha} & 0 & \ldots & o \\
0 & u & \ldots & 0 \\
\vdots & \vdots & \ddots & \vdots \\
0 & 0 & \ldots & u
\end{array}\right),
$$

where $1_{\alpha}$ denotes the unit matrix of order $\alpha$.

Remark. Certainly, $\mathbf{G}:=\mathrm{GL}\left(\infty, \mathbb{Q}_{p}\right)$. But the notation of the type $\mathbf{G}:=$ $\mathrm{GL}\left(\alpha+k \infty, \mathbb{Q}_{p}\right)$ allows us to indicate certain subgroups in $\mathbf{G}$.

We wish to define a structure of a semigroup on double cosets $\mathbf{K} \backslash \mathbf{G} / \mathbf{K}$.

Set

$$
\Theta_{N}:=\left(\begin{array}{ccc}
0 & 1_{N} & 0 \\
1_{N} & 0 & 0 \\
0 & 0 & 1_{\infty}
\end{array}\right) \in \mathbf{K} .
$$

\footnotetext{
${ }^{12}$ There are several non-equivalent non-degenerate quadratic forms and several different orthogonal groups over $\mathbb{Q}_{p}$, however we consider only this group.
} 
Let $\mathfrak{g}, \mathfrak{h} \in \mathbf{K} \backslash \mathbf{G} / \mathbf{K}$. Choose their representatives $g, h \in \mathbf{G}$. Consider the sequence

$$
f_{N}:=g \Im\left(\Theta_{N}\right) h
$$

and double coset $\mathfrak{f}_{N}$ containing $f_{N}$.

Theorem 2.1 a) The sequence $\mathfrak{f}_{N}$ of double cosets is eventually constant.

b) The limit $\mathfrak{f}:=\lim _{N \rightarrow \infty} \mathfrak{f}_{N}$ does not depend on a choice of representatives $g, h$.

c) The product $\mathfrak{g} \star \mathfrak{h}$ in $\mathbf{K} \backslash \mathbf{G} / \mathbf{K}$ obtained in this way is associative.

These statements are simple, see proofs of parallel real statements in [16. Also, it is easy to write an explicit formula for the product. For definiteness, set $k=2$. Then

$$
\begin{aligned}
& \left(\begin{array}{ccc}
a & b_{1} & b_{2} \\
c_{1} & d_{11} & d_{12} \\
c_{2} & d_{21} & d_{22}
\end{array}\right) \star\left(\begin{array}{ccc}
a^{\prime} & b_{1}^{\prime} & b_{2}^{\prime} \\
c_{1}^{\prime} & d_{11}^{\prime} & d_{12}^{\prime} \\
c_{2}^{\prime} & d_{21}^{\prime} & d_{22}^{\prime}
\end{array}\right)= \\
= & \left(\begin{array}{ccccc}
a & b_{1} & 0 & b_{2} & 0 \\
c_{1} & d_{11} & 0 & d_{12} & 0 \\
0 & 0 & 1 & 0 & 0 \\
c_{2} & d_{21} & 0 & d_{22} & 0 \\
0 & 0 & 0 & 0 & 1
\end{array}\right)\left(\begin{array}{cccccc}
1_{\alpha} & 0 & 0 & 0 & 0 \\
0 & 0 & 1_{\infty} & 0 & 0 \\
0 & 1_{\infty} & 0 & 0 & 0 \\
0 & 0 & 0 & 0 & 1_{\infty} \\
0 & 0 & 0 & 1_{\infty} & 0
\end{array}\right)\left(\begin{array}{ccccc}
a^{\prime} & b_{1}^{\prime} & 0 & b_{2}^{\prime} & 0 \\
c_{1}^{\prime} & d_{11}^{\prime} & 0 & d_{12}^{\prime} & 0 \\
0 & 0 & 1 & 0 & 0 \\
c_{2}^{\prime} & d_{21}^{\prime} & 0 & d_{22}^{\prime} & 0 \\
0 & 0 & 0 & 0 & 1
\end{array}\right)
\end{aligned}
$$

Since a result is double coset, we can write the final matrix in different forms, say

or

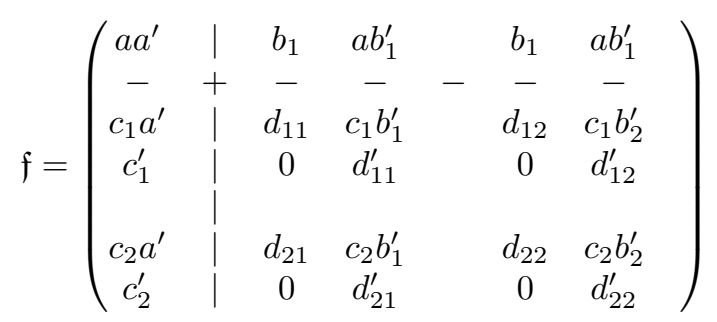

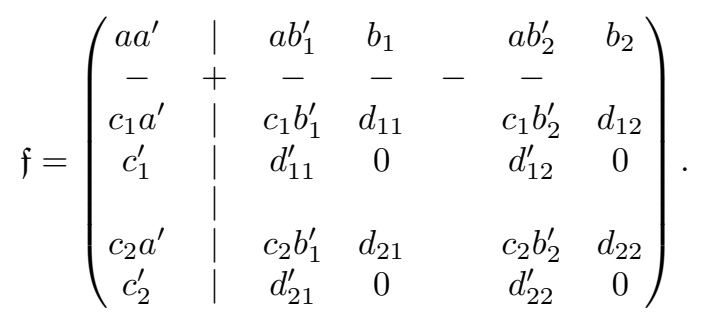

2.3. Multiplicativity theorem. Let $\rho$ be a unitary representation of $\mathbf{G}$, denote by $H^{\mathbf{K}}$ the subspace of all $\mathbf{K}$-fixed vectors. Denote by $P^{\mathbf{K}}$ the operator of orthogonal projection to $H^{\mathbf{K}}$. For $g \in \mathbf{G}$ consider the operator $\bar{\rho}(g): H^{\mathbf{K}} \rightarrow H^{\mathbf{K}}$ given by

$$
\bar{\rho}(g):=\left.P^{\mathrm{K}} \rho(g)\right|_{H^{K}} .
$$


Obviously, $\bar{\rho}(g)$ is a function on double cosets $\mathbf{K} \backslash \mathbf{G} / \mathbf{K}$, therefore we can write $\bar{\rho}(\mathfrak{g})$.

Theorem 2.2 For any unitary representation $\rho$, for all $\mathfrak{g}, \mathfrak{h} \in \mathbf{K} \backslash \mathbf{G} / \mathbf{K}$ the following equality (the "multiplicativity theorem") holds,

$$
\bar{\rho}(\mathfrak{g}) \bar{\rho}(\mathfrak{h})=\bar{\rho}(\mathfrak{g} \star \mathfrak{h}) .
$$

We give a proof in Section 6 .

Remark. Apparently the analog of Proposition 1.12 for $p$-adic case does not hold.

\subsection{Sphericity.}

Proposition 2.3 Let $\alpha=0$. Then the pair $(\mathbf{G}, \mathbf{K})$ is spherical, i.e., for any irreducible unitary representation of $\mathbf{G}$ the dimension of the space of $\mathbf{K}$-fixed vectors is $\leqslant 1$.

We omit a proof, it is the same as for infinite-dimensional real classical groups, see [16].

2.5. Involution. The map $g \mapsto g^{-1}$ induces an involution $\mathfrak{g} \mapsto \mathfrak{g}^{*}$ on $\mathbf{K} \backslash \mathbf{G} / \mathbf{K}$. Evidently,

$$
(\mathfrak{g} \star \mathfrak{h})^{*}=\mathfrak{h}^{*} \star \mathfrak{g}^{*} .
$$

Also, for any unitary representation $\rho$ of $\mathbf{G}$ we have

$$
\bar{\rho}\left(\mathfrak{g}^{*}\right)=\bar{\rho}(\mathfrak{g})^{*} .
$$

2.6. Purpose of the work. Our aim is to describe this multiplication in more usual terms. More precisely, we wish to get $p$-adic analogs of multivariate characteristic functions constructed in [16], 20].

2.7. Structure of the paper. Section 3 contains preliminaries (lattices, Bruhat-Tits buildings, relations, the Weil representation of the Nazarov category). A main construction (characteristic functions of double cosets and their properties) is contained in Section 4. Proofs are given in Section 5.

In Section 6 we prove the multiplicativity theorem. Section 7 contains some constructions of representations. Theorem 7.5 shows a link between the characteristic function and operators $\bar{\rho}(\mathfrak{g})$.

\section{Preliminaries. Submodules, relations, Bruhat- Tits buildings, Nazarov category, and Weil representation}

\section{A. Submodules and convergence}


3.1. Modules. Below the term submodule means an $\mathbb{Z}_{p}$-submodule in a linear space $V=\mathbb{Q}_{p}^{k}$. For each submodule $R \subset \mathbb{Q}_{p}^{k}$ there is a (non-canonical) basis $e_{i} \in \mathbb{Q}_{p}^{k}$ such that

$$
R=\mathbb{Q}_{p} e_{1} \oplus \cdots \oplus \mathbb{Q}_{p} e_{j} \oplus \mathbb{Z}_{p} e_{j+1} \oplus \cdots \oplus \mathbb{Z}_{p} e_{l} .
$$

If $j=k$ then $R$ is a linear subspace. If $j=0, l=k$, then we get a lattice. A formal definition is: a lattice $R$ is a compact $\mathbb{Z}_{p}$-submodule such that $\mathbb{Q}_{p} R=\mathbb{Q}_{p}^{k}$. For details, see, e.g., [1].

Denote by $\operatorname{Mod}(V)$ the set of all submodules in $V$, by Lat $(V)$ the space of all lattices. It is easy to see that

$$
\operatorname{Lat}(V) \simeq \mathrm{GL}\left(V, \mathbb{Q}_{p}\right) / \mathrm{GL}\left(V, \mathbb{Z}_{p}\right) .
$$

For any submodule $R$ denote by $R_{\downarrow}$ the maximal linear subspace in $R$. By $R^{\uparrow}$ we denote the minimal linear subspace containing $R$,

$$
R_{\downarrow} \subset R \subset R^{\uparrow}
$$

The image of $R$ in the quotient space $R^{\uparrow} / R_{\downarrow}$ is a lattice.

Conversely, let $L \subset M$ be a pair of subspaces, $\pi: L \rightarrow L / M$ be the projection. Let $P \subset M / L$ be a lattice. Then $\pi^{-1} P$ is a submodule in $\mathbb{Q}_{p}^{k}$ and all submodules have such form.

3.2. Duality. For a $p$-adic linear space $V$ we denote by $V^{\prime}$ the space of linear functionals on $V$. For a submodule $L \subset V$ define the dual module $L^{\diamond} \subset V^{\prime}$ as the set of all linear functionals $\ell \in V^{\prime}$ such that

$$
\ell(v) \in \mathbb{Z}_{p} \quad \text { for all } v \in L
$$

Notice that $L^{\diamond \diamond}=L$.

If $L$ is a lattice, then $L^{\diamond}$ is a lattice.

3.3. The Hausdorff convergence on $\operatorname{Mod}(V)$. Let $V=\mathbb{Q}_{p}^{n}$. We define a norm on $V$ as

$$
\|x\|=\max _{j}\left|x_{j}\right| .
$$

Denote by $B\left(p^{l}\right)$ the ball with center at 0 of radius $p^{l}$.

Let $K$ be a metric space, $A, B$ be closed subsets. Define the Hausdorff deviation $\eta_{B}(A)$ as the supremum of distance between $a$ ranging in $A$ and $B$ (a number $\eta_{B}(A)$ is a nonnegative real or $\infty$ ). The Hausdorff $\propto 13$ on the space of closed subset is defined by

$$
h(A, B)=\max \left(\eta_{A}(B), \eta_{B}(A)\right) .
$$

Its restriction to the space of compact subsets is a metric. If $K$ is compact then the space of its closed subsets is compact.

\footnotetext{
${ }^{13}$ We allow distance $=+\infty$
} 
Now we introduce the topology on $\operatorname{Mod}(V)$. We say that $R_{j}$ converges to $R$ if for each $l$ we have a convergence $B\left(p^{l}\right) \cap R_{j} \rightarrow B\left(p^{l}\right) \cap R$ in the sense of Hausdorff metric. Notice that this convergence is metrized, a (non-canonical) metric is given by

$$
d(L, M)=\sum_{j=1}^{\infty}(2 p)^{-j} h\left(L \cap B\left(p^{l}\right), M \cap B\left(p^{l}\right)\right) .
$$

Lemma 3.1 a) The space $\operatorname{Mod}(V)$ is compact with respect to the Hausdorff topology.

b) The space $\operatorname{Lat}(V)$ is a discrete dense subset in $\operatorname{Mod}(V)$.

Let us prove a). Choose a convergent subsequence from arbitrary sequence of submodules $L_{j}$. First, we choose a subsequence $L_{j_{k}}$ such that $L_{j_{k}} \cap B\left(p^{0}\right)$ converges. From the latter sequence we choose a subsequence such that intersections with $B\left(p^{1}\right)$ converges. Etc.

3.4. Analog of the radial limit. We need an analog of the radial limit (1.8). Say that a sequence $R_{j}$ of submodules rigidly converges to a submodule $R$ (notation $R_{j} \nearrow R$ ) if

(A) for any compact subset $S \subset R$ we have $S \subset R_{j}$ starting some place.

(B) for each $\varepsilon>0$, for sufficiently large $j$ the set $R_{j}$ is contained in the $\varepsilon$-neighborhood of $R$.

ExAmPle. Let $V=\mathbb{Q}_{p}^{2}$. Let $R_{j}=p^{-k} \mathbb{Z}_{p} e_{1} \oplus p^{k} \mathbb{Z}_{p} e_{2}$. Then $R_{j}$ rigidly converges to a line $\mathbb{Q}_{p} e_{1}$. Now let

$$
S_{j}=\mathbb{Z}_{p}\left(p^{-k} e_{1}+e_{2}\right) \oplus p^{k} \mathbb{Z}_{p} e_{2} .
$$

Then $S_{j}$ converges to the line $\mathbb{Q}_{p} e_{1}$ in Hausdorff sense but not rigidly.

Evidently, we can reformulate the condition (A) as

$$
\eta_{R}\left(R_{j}\right) \rightarrow 0
$$

Lemma 3.2 The condition (B) is equivalent to

$$
\eta_{R \diamond}\left(R_{j}^{\diamond}\right) \rightarrow 0 .
$$

Proof. Let us equip $V^{\prime}$ by the dual norm. Let $S, S_{j} \in V^{\prime}$ and $\eta_{S}\left(S_{j}\right) \rightarrow 0$. For small $\varepsilon>0$ we have

$$
S_{j} \subset S+B(\varepsilon) .
$$

Passing to the duals, we get

$$
S_{j}^{\diamond} \supset S^{\diamond} \cap B\left(\varepsilon^{-1}\right) .
$$

But $S^{\diamond} \cap B\left(\varepsilon^{-1}\right)$ is an exhausting sequence of compact subsets in $S^{\diamond}$. Also, (3.3) implies (3.2). 
Lemma 3.3 If $R_{j} \nearrow R$, then $\left(R_{j}\right)_{\downarrow} \subset R_{\downarrow}$ and $\left(R_{j}\right)^{\uparrow} \supset R^{\uparrow}$ starting some $j$.

Proof. The first claim. For sufficiently large $k$ we have $R \subset R_{\downarrow}+B\left(p^{k}\right)$, also $B\left(p^{k}\right)+B(\varepsilon)=B\left(p^{k}\right)$ for $\varepsilon \leqslant p^{k}$. Therefore for a large $j$ we have

$$
R_{\downarrow}+B\left(p^{k}\right) \supset R_{j} \supset\left(R_{j}\right)_{\downarrow}
$$

But a subspace, which is contained in a tube neighborhood of a subspace $R_{\downarrow}$, is contained in $R_{\downarrow}$.

The second claim. We consider a compact subset $K \subset R$ generating $R^{\uparrow}$ as a $\mathbb{Q}_{p}$-subspace. Then $\left(R_{j}\right)^{\uparrow}$ contains $K$ for sufficiently large $j$ and therefore $\left(R_{j}\right)^{\uparrow} \supset R^{\uparrow}$.

In particular, a $\nearrow$-convergent sequence of linear subspaces is eventually constant.

Lemma 3.4 a) Let $L \subset V$ be a linear subspace. If $R_{j} \nearrow R$, then $\left(L \cap R_{j}\right) \nearrow$ $(L \cap R)$.

b) Let $M \subset V$ be a linear subspace, denote by $\pi$ the natural map $V \rightarrow V / M$. If $R_{j} \nearrow R$ then $\pi\left(R_{j}\right) \nearrow \pi(R)$.

Proof. a) Only condition (B) requires a proof, i.e., for each $\varepsilon>0$ there exists $N$ such that for $j \geqslant N$

$$
R_{j} \cap L \subset(R \cap L)+B(\varepsilon) .
$$

It is easy to shown that there is a basis $e_{m}$ in $\mathbb{Q}_{p}^{n}$ such that $R$ has canonical form (3.1) and $L$ is a linear span of several basis elements. Then for sufficiently big $N$ we have

$$
\left(R+p^{N} \oplus \mathbb{Z}_{p} e_{j}\right) \cap L \subset(R \cap L)+p^{N} \oplus \mathbb{Z}_{p} e_{j} .
$$

Passing from the basis $e_{m}$ to the standard basis in $\mathbb{Q}_{p}^{n}$ we get

$$
(R+B(\delta)) \cap L \subset(R \cap L)+B(C \delta) .
$$

where $C=C(R, L)$ is a constant. Now we take $\delta=\varepsilon / C$ and choose number $k$, starting which $R_{j} \subset R+B(\delta)$.

b) follows from a) by the duality.

REMARK. $\nearrow$-convergence is not metrizable.

\section{B. Bruhat-Tits buildings}

3.5. Self-dual modules. For details, see [9], Sections 10.6-10.7. Consider a $p$-adic linear space $V \simeq \mathbb{Q}_{p}^{2 n}$ equipped with a nondegenerate skew-symmetric bilinear form $B_{V}(\cdot, \cdot)$ (as above). We say that a subspace $L$ is isotropic if $B_{V}(v, w)=0$ for all $v, w \in V$. By $\operatorname{LGr}(V)$ we denote the set of all maximal isotropic (Lagrangian) subspaces in $V($ their dimensions $=n)$. 
By $L^{\perp}$ we denote the ortho-dual of a subspace $L$, i.e set of all vectors $w$ such that $B_{V}(v, w)=0$ for all $v \in L$.

If $P$ is a submodule, denote by $P^{\Perp}$ the dual submodule, i.e., the set of vectors $w$ such that $B(v, w) \in \mathbb{Z}_{p}$ for all $v \in P$. If $P$ is a subspace, then $P^{\Perp}=P^{\perp}$.

We say that a submodule $R \subset V$ is isotropic if $B_{V}(v, w) \in \mathbb{Z}_{p}$ for all $v$, $w \in R$.

ExAmple. If $R$ is a linear subspace, then $R$ is isotropic in the usual sense. On the other hand, the lattice $\mathbb{Z}_{p}^{2 n}$ is isotropic (and self-dual, see below).

We say that a submodule $R$ is self-dual if it is a maximal isotropic submodule in $V$. Equivalently, $P^{\Perp}=P$. Denote by $\operatorname{LMod}(V)$ the set of all self-dual submodules in $V$, by LLat $(V)$ the set of all self-dual lattices. It is easy to show that $\operatorname{Sp}\left(2 n, \mathbb{Q}_{p}\right)$ acts on $\operatorname{LLat}(V)$ transitively and

$$
\operatorname{LLat}(V)=\operatorname{Sp}\left(2 n, \mathbb{Q}_{p}\right) / \operatorname{Sp}\left(2 n, \mathbb{Z}_{p}\right) .
$$

Lemma 3.5 a) For any self-dual submodule $R$ the subspace $R_{\downarrow}$ is isotropic, and $R^{\uparrow}$ is the ortho-dual of $R_{\downarrow}$.

b) Let $L$ ranges in the set of isotropic subspaces. Denote by $\pi: L^{\perp} \rightarrow L^{\perp} / L$ the natural projection map. Any self-dual submodule $R$ has the form $\pi^{-1} S$, where $S$ is a self-dual lattice in $L^{\perp} / L$.

c) The unique $\operatorname{Sp}(V)$-invariant of a self-dual module $R$ is $\operatorname{dim} R_{\downarrow}$.

These statement is obvious.

Sometimes it is convenient to reformulate a definition of an isotropic module. Define a bicharacter $\beta$ on $V \times V$ by

$$
\beta(x, y)=\exp \{2 \pi i B(x, y)\} .
$$

We say that a module $P$ is isotropic if $\beta(x, y)=1$ on $P \times P$.

3.6. Almost self-dual modules. Let $V$ and $B$ be same as above. A submodule $L$ in $V$ is almost self-dual if it contains a self-dual module $M$ and $B(v, w) \in p^{-1} \mathbb{Z}_{p}$ for all $v, w \in L$ (see, e.g., [8], Section 10.6). Notice that $L / M \simeq(\mathbb{Z} / p \mathbb{Z})^{k}$ with $k=0,1, \ldots, n$.

Lemma 3.6 a) Any almost self-dual module can be reduced by a symplectic transformation to the form

$$
\begin{aligned}
\left(p^{-1} \mathbb{Z}_{p} e_{1} \oplus \mathbb{Z}_{p} e_{n+1}\right) \oplus \cdots \oplus\left(p^{-1} \mathbb{Z}_{p} e_{k} \oplus \mathbb{Z}_{p} e_{n+k}\right) \oplus & \\
\oplus\left(\mathbb{Z}_{p} e_{k+1} \oplus \mathbb{Z}_{p} e_{n+k+1}\right) \oplus \cdots \oplus\left(\mathbb{Z}_{p} e_{m} \oplus \mathbb{Z}_{p} e_{n+m}\right) \oplus & \oplus \mathbb{Q}_{p} e_{m+1} \oplus \cdots \oplus \mathbb{Q}_{p} e_{n}
\end{aligned}
$$

b) The only $\operatorname{Sp}(V)$-invariants of an almost self-adjoint module $R$ are $\operatorname{dim} R_{\downarrow}$ and the number $k$ (rank of an Abelian group $R / S$, where $S$ is a self-dual submodule in $R$. For almost self-dual lattices the only $\operatorname{Sp}(V)$-invariant is the volume of $R$, it is equal $p^{-k}$. 
3.7. Graph $\Delta(V)$. Consider a $p$-adic linear space $V$ equipped with a nondegenerate skew-symmetric bilinear form $B$ as above. We draw an oriented graph $\Delta(V)$. Vertices are almost self-dual modules in $V$. If $R \supset R^{\prime}$, then we draw an arrow from $R$ to $R^{\prime}$.

If $R, R^{\prime}$ are connected by an arrow, then $R_{\downarrow}=\left(R^{\prime}\right)_{\downarrow}$ and $R^{\uparrow}=\left(R^{\prime}\right)^{\uparrow}$.

Any pair of lattices can be connected by a (non-oriented) way. Denote the subgraph whose vertices are all lattices by $\Delta_{0}(V)$.

More generally, fix an isotropic subspace $L$ and consider the subgraph $\Delta_{L}(V)$ whose vertices are almost self-dual modules $R$ such that $R_{\downarrow}=L, R^{\uparrow}=L^{\perp}$. We get a connected subgraph, moreover

$$
\Delta_{L}(V) \simeq \Delta_{0}\left(L^{\perp} / L\right) .
$$

By definition,

$$
\Delta(V)=\bigsqcup_{L \text { is isotropic subspace }} \Delta_{L}(V) .
$$

If $L \subset M$, then $\Delta_{M}$ is contained in the closure of $\Delta_{L}$ in the sense of $\nearrow_{-}$ convergence.

3.8. Bruhat-Tits buildings, for details, see [42, 8. Now we consider all $k$-plets of vertices of $\Delta(V)$ that are pairwise connected by edges. For any such $k$-plet we draw a $(k-1)$-simplex with given vertices and edges. Faces of a simplex correspond to subsets of the $k$-plet. Thus we get a simplicial complex, denote it by $\operatorname{Bd}(V)$.

Consider the subgraph $\Delta_{0}$. It can be shown that $k \leqslant n+1$ and each simplex is contained in an $n$-dimensional simplex. In this way we get a structure of an $n$-dimensional simplicial complex, it is called a Bruhat-Tits building. We denote it by $\operatorname{Bd}_{0}(V)$.

For a subgraph $\Delta_{L}$ we get a simplicial complex complex $\mathrm{Bd}_{L}(V)$ isomorphic $\operatorname{Bd}\left(L^{\perp} / L\right)$.

Below we use term 'distinguished boundary of a building' for the Lagrangian Grassmannian, this is an counterpart of Shilov boundary.

\section{Relations and Nazarov category}

3.9. Relations. Let $V, W$ be linear spaces. We say that a relation $P$ : $V \rightrightarrows W$ is a submodule in $V \oplus W$.

ExAmple. Let $A: V \rightarrow W$ be a linear operator. Then its graph is a relation.

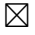

Let $P: V \rightrightarrows W, Q: W \rightrightarrows Y$ be relations. We define their product $S=Q P: V \rightrightarrows Y$ as the set of all $v \oplus y \in V \oplus Y$ for which there exists $w \in W$ such that $v \oplus w \in P, w \oplus y \in Q$.

For a relation $P: V \rightrightarrows W$ we define its kernel ker $P \subset V$ as

$$
\text { ker } P=P \cap(V \oplus 0) \text {, }
$$




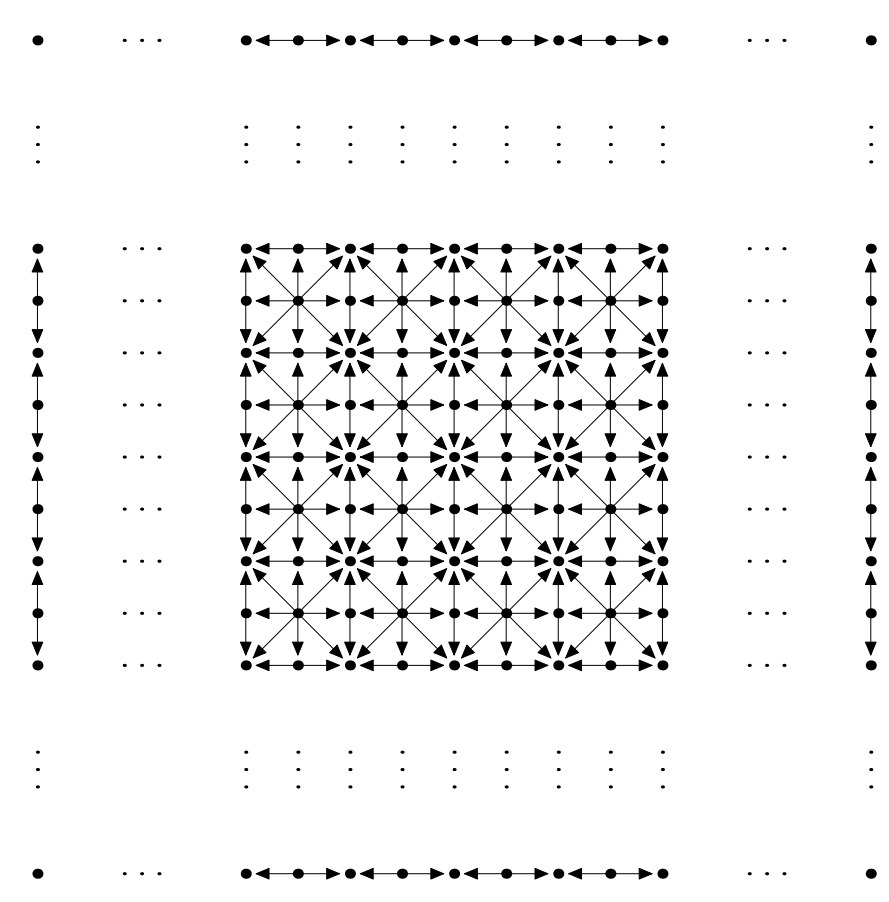

Figure 1: A reference to Subsections 3.4 3.6. A subcomplex ('apartment') of the building $\operatorname{Bd}\left(\mathbb{Q}_{p}^{4}\right)$ corresponding to lattices of the form $R_{1} \oplus \cdots \oplus R_{4}$, where $R_{j}$ is a submodule in the line $\mathbb{Q}_{p} e_{j}$.

1) Vertices of the central piece of the subcomplex are almost self-dual lattices of the form $L=p^{k_{1}} \mathbb{Z}_{p} e_{1} \oplus p^{k_{2}} \mathbb{Z}_{p} e_{2} \oplus p^{l_{1}} \mathbb{Z}_{p} e_{3} \oplus p^{l_{2}} \mathbb{Z}_{p} e_{4}$. They are almost self-dual iff $k_{1}+l_{1}, k_{2}+l_{2}$ are 0 or -1 .

2) Four boundary pieces. Each piece corresponds to almost self-dual submodules containing a line $\mathbb{Q}_{p} e_{j}$, where $j=1,2,3,4$. For instance, for $j=1$ such submodules have a form $M=\mathbb{Q}_{p} e_{1} \oplus p^{m_{2}} \mathbb{Z}_{p} e_{2} \oplus p^{l_{2}} \mathbb{Z}_{p} e_{4}$, where $m_{2}+l_{2}=0$ ,1. A sequence of lattices $\nearrow$-converges to $M$ only if $k_{1} \rightarrow-\infty$ and $k_{2}=m_{2}$ starting some place.

3) Four extreme points correspond to Lagrangian planes spanned by pairs of vectors $\left(e_{1}, e_{2}\right),\left(e_{1}, e_{4}\right),\left(e_{2}, e_{3}\right),\left(e_{3}, e_{4}\right)$. A sequence of lattices $\nearrow$-converges to $\mathbb{Q}_{p} e_{1} \oplus \mathbb{Q}_{p} e_{4}$ iff $k_{1} \rightarrow+\infty, k_{2} \rightarrow-\infty$. 
the indefiniteness indef $P \subset W$,

$$
\text { indef } P=P \cap(0 \oplus W),
$$

the domain of definiteness

$$
\operatorname{dom} P=\text { projection of } P \text { to } V \text {, }
$$

and the image

$$
\operatorname{im} P=\text { projection of } P \text { to } W \text {. }
$$

We define the pseudo-inverse relation $P^{\square}: W \rightrightarrows V$ being the same submodule in $W \oplus V \simeq V \oplus W$. Evidently,

$$
(P Q)^{\square}=Q^{\square} P^{\square} .
$$

3.10. The definition of product. A reformulation. We keep the same notation. Consider the space $\mathcal{Z}:=V \oplus W \oplus W \oplus Y$ and following submodules of $\mathcal{Z}$ :

— the subspace $\mathcal{H}$ consisting of vectors $v \oplus w \oplus w \oplus y$;

— the subspace $\mathcal{A}$ consisting of vectors $0 \oplus w \oplus w \oplus 0$;

- the submodule $P \oplus Q \subset(V \oplus W) \oplus(W \oplus Y)$.

Then we do the following operations:

- take the intersection $R=\mathcal{H} \cap(P \oplus Q)$;

— take the map $\theta: \mathcal{H} \rightarrow \mathcal{H} / \mathcal{A} \simeq V \oplus Y$.

Then $Q P=\theta(R)$.

3.11. Action on $\operatorname{Mod}(V)$. Let $P: V \rightrightarrows W$ be a relation, $T$ be a submodule in $V$. We define the submodule $P T \subset W$ as the set of $w \in W$ such that there is $v \in T$ satisfying $v \oplus w \in P$.

REMARK. We can consider a submodule $T \subset V$ as a relation $0 \rightrightarrows V$. Therefore we can regard $P T: 0 \rightrightarrows W$ as the product of relations $T: 0 \rightrightarrows V$ and $Q: V \rightrightarrows W$.

3.12. The Nazarov category. For a pair $V, W$ of symplectic linear spaces we define a skew-symmetric bilinear form $B^{\ominus}$ on $V \oplus W$ by

$$
B^{\ominus}\left(v \oplus w, v^{\prime} \oplus w^{\prime}\right)=B_{V}\left(v, v^{\prime}\right)-B_{W}\left(w, w^{\prime}\right) .
$$

Denote by

- $\overline{\operatorname{Naz}}(V, W)$ the set of all self-dual submodules of $V \oplus W$;

- $\operatorname{Naz}(V, W)$ the set of $P \in \overline{\mathrm{Naz}}(V, W)$ such that ker $P$ and indef $P$ are compact.

Theorem 3.7 Let $P \in \overline{\operatorname{Naz}}(V, W)$, let $T$ be a self-dual submodule in $V$. Then the submodule $P T \subset W$ is self-dual. 
In [9], Theorem 10.7.2, the same statement is established under slightly stronger condition $P \in \operatorname{Naz}(V, W)$. In fact, a proof remains valid for $P \in$ $\overline{\mathrm{Naz}}(V, W)$.

Theorem 3.8 a) If $P \in \operatorname{Naz}(V, W), Q \in \operatorname{Naz}(W, Y)$, then $Q P \in \operatorname{Naz}(V, Y)$.

b) If $P \in \overline{\mathrm{Naz}}(V, W), Q \in \overline{\mathrm{Naz}}(W, Y)$, then $Q P \in \overline{\mathrm{Naz}}(V, Y)$.

c) If $P \in \operatorname{Naz}(V, W), Q \in \operatorname{Naz}(W, Y)$ are lattices, then $Q P$ is a lattice.

The statement a) was proved in Nazarov [33] (see also [9], Section 10.7), c) is obvious. The statement b) is a corollary of Theorem 3.7, see [9, Subsection 10.7.4.

Thus we get two similar categorie 14 , Naz and $\overline{\mathrm{Naz}}$. The group of automorphisms of an object $V$ is the symplectic group $\operatorname{Sp}\left(V, \mathbb{Q}_{p}\right)$ (for both categories), an operator $V \rightarrow V$ is symplectic iff its graph is isotropic with respect to the form $B^{\ominus}$.

For $P \in \overline{\mathrm{Naz}}(V, W)$, we have

$$
\begin{array}{ll}
(\operatorname{ker} P)^{\Perp}=\operatorname{dom} P, & (\text { indef } P)^{\Perp}=\operatorname{im} P \\
\left((\operatorname{ker} P)_{\downarrow}\right)^{\perp}=(\operatorname{dom} P)^{\uparrow}, & \left((\text { indef } P)_{\downarrow}\right)^{\Perp}=(\operatorname{im} P)^{\uparrow},
\end{array}
$$

\subsection{Action of the Nazarov category on buildings.}

Proposition 3.9 a) Let $P \in \operatorname{Naz}(V, W)$, let $T$ be an almost-self-dual lattice. Then $P T \subset W$ is an almost self-dual lattice.

b) Let $P \in \overline{\mathrm{Naz}}(V, W)$, let $T$ be an almost-self-dual submodule. Then $P T \subset$ $W$ is an almost self-dual submodule.

The statement a) is 9], Proposition 10.7.5, a proof remains to be valid for the statement b) also.

Now, let $\Xi, \Sigma$ be simplicial complexes, let $\operatorname{Vert}(\Xi)$, Vert $(\Sigma)$ be their sets of vertices. We say, that a map $15 \operatorname{Vert}(\Xi) \rightarrow \operatorname{Vert}(\Sigma)$ is simplicial, if for any simplex $\Delta \subset \Xi$ images of its vertices are are contained in one simplex of $\Sigma$. Notice, that we can extend a simplicial map to a map of complexes $\Xi \rightarrow \Sigma$ assuming that a map is affine on each face.

The following statement is a corollary of Proposition 3.9.

Theorem 3.10 a) A morphism $P \in \operatorname{Naz}(V, W)$ induces simplicial map

$$
\operatorname{Bd}(V) \rightarrow \operatorname{Bd}(W)
$$

sending

$$
\mathrm{Bd}_{0}(V) \rightarrow \mathrm{Bd}_{0}(W) .
$$

\footnotetext{
${ }^{14}$ The Nazarov category is an analog of Krein-Shmulian type categories, see [8], 9]

15 generally, non-injective.
} 
b) $A$ morphism $P \in \overline{\mathrm{Naz}}(V, W)$ induces a simplicial map $\mathrm{Bd}(V) \rightarrow \mathrm{Bd}(V)$, sending $\operatorname{Bd}(V)$ to

$$
\operatorname{Bd}(V) \rightarrow \bigsqcup_{M \text { is isotropic subspace in } W} \quad \operatorname{Bd}\left[M^{\perp} / M\right] .
$$

REMARK. The map $T \rightarrow P T$ is contractive in an essentially stronger sense, see 43 .

Theorem 3.11 Let $P \in \overline{\mathrm{Naz}}(V, W)$. The the induced map $\mathrm{Bd}(V) \rightarrow \operatorname{Bd}(W)$ is $\nearrow$-continuous, i.e., for a convergent sequence $T_{j} \nearrow T$ of almost self-dual modules, we have $P T_{j} \nearrow P T$.

Proof. We evaluate $P T_{j}$ according procedure described in Subsection 3.10 By Lemma 3.4. both steps of the evaluation are continuous.

\section{Weil representation}

The Weil representation is used below only in Section 7 .

3.14. Extended Nazarov category. Now we add to the Nazarov category an infinite-dimensional object $V_{2 \infty}$. This is the space of vectors

$$
\left(x_{1}^{+}, x_{2}^{+}, \ldots, x_{1}^{-}, x_{2}^{-}, \ldots\right), \quad \text { where } x_{j}^{ \pm} \in \mathbb{Q}_{p} \text { and } x_{j}^{ \pm} \in \mathbb{Z}_{p} \text { for almost all } j .
$$

Notice that $V_{2 \infty}$ is not a $\mathbb{Q}_{p}$-linear space but is a $\mathbb{Z}_{p}$-module.

We introduce a bicharacter $\beta(\cdot, \cdot)$ on $V_{2 \infty} \oplus V_{2 \infty}$ by

$$
\beta(x, y)=\exp \left[2 \pi i \sum_{j=1}^{\infty}\left(x_{j}^{+} y_{j}^{-}-x_{j}^{-} y_{j}^{+}\right)\right]:=\prod_{j=1}^{\infty} \exp \left\{2 \pi i\left(x_{j}^{+} y_{j}^{-}-x_{j}^{-} y_{j}^{+}\right)\right\} .
$$

Notice that almost all factors of the product equal to 1 . The sum in square brackets defining a symplectic form is not well defined, more precisely it is well defined modulo $\mathbb{Z}_{p}$.

Objects of the extended Nazarov category $\mathbf{N a z}$ are

- finite-dimensional spaces $V$ equipped with skew-symmetric non-degenerate bilinear forms $B_{V}$ and with the corresponding bicharacters $\beta_{V}$, see (3.4);

- the space $V_{2 \infty}$.

Let $V, W$ be two objects. We equip their direct sum with a bicharacter

$$
\beta_{V \oplus W}\left(v \oplus w, v^{\prime} \oplus w^{\prime}\right)=\frac{\beta_{V}\left(v, v^{\prime}\right)}{\beta_{W}\left(w, w^{\prime}\right)} .
$$

A morphism of the category $\mathbf{N a z}$ is a self-dual submodule $P \subset V \oplus W$ such that ker $P$ and indef $P$ are compact. 
Group $\mathbf{S p}\left(2 \infty, \mathbb{Q}_{p}\right)$ of automorphisms of $V_{2 \infty}$ consists of $2 \infty \times 2 \infty$ matrices $r=\left(\begin{array}{ll}a & b \\ c & d\end{array}\right)$ such that

- all but a finite number of matrix elements are integer;

- matrix elements $a_{i j}, b_{i j}, c_{i j}, d_{i j}$ tend to 0 as $i \rightarrow \infty$ for fixed $j$; also they tend to 0 as $j \rightarrow \infty$ for fixed $i$;

- matrices $r$ are symplectic in the usual sense,

$$
r^{t}\left(\begin{array}{cc}
0 & 1 \\
-1 & 0
\end{array}\right) r=\left(\begin{array}{cc}
0 & 1 \\
-1 & 0
\end{array}\right)=r\left(\begin{array}{cc}
0 & 1 \\
-1 & 0
\end{array}\right) r^{t}
$$

3.15. Heisenberg groups. For the sake of simplicity, set $p>2$. Denote by $\mathbb{T}_{p} \subset \mathbb{C}^{\times}$the group of complex roots of unity of degrees $p, p^{2}, p^{3}, \ldots$ Let $V$ be an object of the extended Nazarov category. We define the Heisenberg group Heis $(V)$ as a central extension of the Abelian group $V$ by $\mathbb{T}_{p}$ in the following way. As a set, $\operatorname{Heis}(V) \simeq V \times \mathbb{T}_{p}$. The multiplication is given by

$$
(v, \lambda) \cdot(w, \mu)=\left(v+w, \lambda \mu \cdot \beta_{V}(v, w)\right) .
$$

Decompose $V=V_{+} \oplus V_{-}$as in (2.1). For a finite dimensional $V$ we define a unitary representation $\Psi$ of $\operatorname{Heis}(V)$ in $L^{2}\left(\mathbb{Q}_{p}^{n}\right)$ by the formula

$$
\Psi\left(v^{+} \oplus v^{-}, \lambda\right) f(x)=\lambda f\left(x+v^{+}\right) \exp \left\{2 \pi i\left(\sum v_{j}^{+} x_{j}+\frac{1}{2} \sum v_{j}^{+} v_{j}^{-}\right)\right\} .
$$

Next, consider the space $\mathcal{E}_{\infty}$ consisting of sequences $z=\left(z_{1}, z_{2}, \ldots\right)$ such that $\left|z_{j}\right| \leqslant 1$ for all but a finite number of $j$. This space is an Abelian locally compact group, it admits a Haar measure. On the open subgroup $\mathbb{Z}_{p}^{\infty} \subset \mathcal{E}_{\infty}$, the Haar measure is a product of probability Haar measures on $\mathbb{Z}_{p}$. The whole space $\mathcal{E}_{\infty}$ is a countable disjoint union of sets $u+\mathbb{Z}_{p}^{\infty}$.

We define the representation of the group $\operatorname{Heis}\left(V_{2 \infty}\right)$ in $L^{2}\left(\mathcal{E}_{\infty}\right)$ by the same formula (3.7).

3.16. The Weil representation of the Nazarov category. Formal definition. See [33, 34, for finite-dimensional case, see [9], Chapter 11.

Theorem 3.12 For a $2 n$-dimensional object of the category $\mathbf{N a z}$ we assign the Hilbert space $\mathcal{H}(V):=L^{2}\left(\mathbb{Q}_{p}^{n}\right)$. For the object $V_{2 \infty}$, we assign the Hilbert space $\mathcal{H}\left(V_{2 \infty}\right):=L^{2}\left(\mathcal{E}_{\infty}\right)$.

a) Let $V, W$ be objects of Naz. Let $P: V \rightrightarrows W$ be a morphism of category Naz. Then there is a unique up to a scalar factor bounded operator

$$
\mathrm{We}(P): \mathcal{H}(V) \rightarrow \mathcal{H}(W)
$$

such that

$$
\Psi(w, 1) \mathrm{We}(P)=\mathrm{We}(P) \Psi(v, 1) \quad \text { for all } v \oplus w \in P \text {. }
$$


b) Let $V, W, Y$ be objects of Naz. Let $P: V \rightrightarrows W, Q: W \rightrightarrows Y$ be morphisms of Naz. Then

$$
\mathrm{We}(Q) \mathrm{We}(P)=s \cdot \mathrm{We}(Q P),
$$

where $s=s(P, Q) \in \mathbb{C}^{\times}$is a nonzero scalar. In other words, we get a projective representation of the category Naz. Also,

$$
\operatorname{We}\left(P^{\square}\right)=t \cdot \operatorname{We}(P)^{*}, \quad t \in \mathbb{C}^{\times} .
$$

For symplectic groups $\operatorname{Sp}\left(2 n, \mathbb{Q}_{p}\right)=\operatorname{Aut}\left(\mathbb{Q}_{p}^{2 n}\right)$ the representation We $(g)$ coincides with the Weil representation.

3.17. Explicit formulas for operators for some morphisms.

1) Let $V=W$ and $P$ be a graph of a symplectic operator. There are simple formulas for some special symplectic matrices:

$$
\begin{aligned}
\text { We }\left(\begin{array}{cc}
A & 0 \\
0 & A^{t-1}
\end{array}\right) f(z) & =|\operatorname{det} A|^{1 / 2} f(z A) \\
\text { We }\left(\begin{array}{cc}
1 & B \\
0 & 1
\end{array}\right) f(z) & =\exp \left\{\pi i z B z^{t}\right\} \\
\mathrm{We}\left(\begin{array}{cc}
0 & 1 \\
-1 & 0
\end{array}\right) f(z) & =\int_{\mathbb{Q}_{p}^{n}} f(x) \exp \left\{2 \pi i x z^{t}\right\} d x .
\end{aligned}
$$

Any element of $\operatorname{Sp}\left(2 n, \mathbb{Q}_{p}\right)$ can be represented as a product of matrices of such forms, this allows to write an explicit formula for $\mathrm{We}(g)$ for any element $g \in$ $\operatorname{Sp}\left(2 n, \mathbb{Q}_{p}\right)$.

Denote by $I(x)$ the function on $\mathbb{Q}_{p}$ defined by

$$
I(x)= \begin{cases}1, & |x| \leqslant 1 \\ 0, & \text { otherwise }\end{cases}
$$

Next, we need some special non-invertible morphisms.

2) Let $V=\mathbb{Q}_{p}^{2 n}, W=V \oplus Y$, where $Y=\mathbb{Q}_{p}^{2 n}$ or $V_{2 \infty}$. Denote by $Y\left(\mathbb{Z}_{p}\right)$ the lattice $\mathbb{Z}_{p}^{2 n}$ or $\mathbb{Z}_{p}^{2 \infty}$ respectively. Denote by

$$
\lambda_{W}^{V}: V \rightrightarrows W
$$

the direct sum of the graph graph $\left(1_{V}\right)$ of the unit operator $1_{V}: V \rightarrow V$ and the lattice $Y\left(\mathbb{Z}_{p}\right) \subset Y$. Then

$$
\mathrm{We}\left(\lambda_{W}^{V}\right) f\left(v_{1}, \ldots, v_{n}, y_{1}, y_{2}, \ldots\right)=f\left(v_{1}, \ldots, v_{n}\right) I\left(y_{1}\right) I\left(y_{2}\right) \ldots
$$

3) Preserving the previous notation denote by

$$
\theta_{W}^{V}: W \rightrightarrows W
$$


the direct sum

$$
\operatorname{graph}\left(1_{V}\right) \oplus\left(Y\left(\mathbb{Z}_{p}\right) \oplus Y\left(\mathbb{Z}_{p}\right)\right) \subset(V \oplus V) \oplus(Y \oplus Y) .
$$

Then

$$
\theta_{W}^{V}=\lambda_{W}^{V}\left(\lambda_{W}^{V}\right)^{*}, \quad\left(\theta_{W}^{V}\right)^{2}=\theta_{W}^{V}, \quad\left(\lambda_{W}^{V}\right)^{*} \lambda_{W}^{V}=1_{V} .
$$

The operator $\operatorname{We}\left(\theta_{W}^{V}\right)$ is the orthogonal projection to the space of functions of the form

$$
f\left(v_{1}, \ldots, v_{n}\right) I\left(y_{1}\right) I\left(y_{2}\right) \ldots
$$

3.18. General case. Any morphism of the category Naz can be represented as a product of morphisms of the types described above. Moreover, for finite dimensional $V, W$, any $P: V \rightrightarrows W$ can be represented as

$$
P=\left(\lambda_{Z}^{W}\right)^{*} \cdot g \cdot \lambda_{Z}^{V}, \quad g \in \operatorname{Sp}(Z),
$$

where $Z$ is sufficiently large $(\operatorname{dim} Z \geqslant 2 \max (\operatorname{dim} V, \operatorname{dim} W))$. In fact, the same decomposition holds for morphisms $Q: V_{2 \infty} \rightarrow V_{2 \infty}$, any $Q$ can be represented as

$$
Q=\theta_{V_{2 \infty} \oplus V_{2 \infty}}^{V_{2 \infty}} \cdot g \cdot \theta_{V_{2 \infty} \oplus V_{2 \infty}}^{V_{2 \infty}}, \quad g \in \operatorname{Sp}\left(V_{2 \infty} \oplus V_{2 \infty}\right) .
$$

\section{Characteristic function}

Here we define characteristic functions of double cosets $\mathbf{K} \backslash \mathbf{G} / \mathbf{K}$ and formulate several theorems. Proofs are in the next section.

4.1. Construction. Consider the group

$$
\mathrm{GL}\left(\alpha+k \infty, \mathbb{Q}_{p}\right):=\lim _{j \rightarrow \infty} \mathrm{GL}\left(\alpha+k j, \mathbb{Q}_{p}\right) .
$$

Let $g \in \mathrm{GL}\left(\alpha+k \infty, \mathbb{Q}_{p}\right)$ actually be contained in $\mathrm{GL}\left(\alpha+k m, \mathbb{Q}_{p}\right)$,

$$
g=\left(\begin{array}{cccc}
a & b_{1} & \ldots & b_{k} \\
c_{1} & d_{11} & \ldots & d_{1 k} \\
\vdots & \vdots & \ddots & \vdots \\
c_{k} & d_{k 1} & \ldots & d_{k k}
\end{array}\right) \in \mathrm{GL}\left(\alpha+k m, \mathbb{Q}_{p}\right) .
$$

We write the following equation (this is an analog of (1.5), the analogy is important)

$$
\left(\begin{array}{c}
v^{+} \\
y_{1}^{+} \\
\vdots \\
y_{k}^{+} \\
v^{-} \\
y_{1}^{-} \\
\vdots \\
y_{k}^{-}
\end{array}\right)=\left(\begin{array}{cccccccc}
a & b_{1} & \ldots & b_{k} & 0 & 0 & \ldots & 0 \\
c_{1} & d_{11} & \ldots & d_{1 k} & 0 & 0 & \ldots & 0 \\
\vdots & \vdots & \ddots & \vdots & \vdots & \vdots & \ddots & \vdots \\
c_{k} & d_{k 1} & \ldots & d_{k k} & 0 & 0 & \ldots & 0 \\
0 & 0 & \ldots & 0 \\
0 & 0 & \ldots & 0 \\
\vdots & \vdots & \ddots & \vdots \\
0 & 0 & \ldots & 0
\end{array} \quad\left(\begin{array}{cccc}
a & b_{1} & \ldots & b_{k} \\
c_{1} & d_{11} & \ldots & d_{1 k} \\
\vdots & \vdots & \ddots & \vdots \\
c_{k} & d_{k 1} & \ldots & d_{k k}
\end{array}\right)\left(\begin{array}{c}
u^{+} \\
x_{1}^{+} \\
\vdots \\
x_{k}^{+} \\
u^{-} \\
x_{1}^{-} \\
\vdots \\
x_{k}^{-}
\end{array}\right) .\right.
$$


Here $u^{ \pm}, v^{ \pm} \in \mathbb{Q}_{p}^{\alpha}$ and $x_{j}^{ \pm}, y_{j}^{ \pm} \in \mathbb{Q}_{p}^{m}$.

Before the exploring of this identity as (1.5), we need some preparations.

Define 3 spaces, $\mathcal{V}, \mathcal{H}, \ell_{m}$ :

1) Denote $\mathcal{V}:=\mathbb{Q}_{p}^{\alpha} \oplus \mathbb{Q}_{p}^{\alpha}$. We regard $u=u^{+} \oplus u^{-}, v=v^{+} \oplus v^{-}$as elements of $\mathcal{V}$. Equip $\mathcal{V}$ with the standard skew-symmetric bilinear form $\left(\begin{array}{cc}0 & 1 \\ -1 & 0\end{array}\right)$.

2) Denote

$$
\mathcal{H}:=\mathcal{H}^{+} \oplus \mathcal{H}^{-}=\mathbb{Q}_{p}^{k} \oplus \mathbb{Q}_{p}^{k}
$$

and equip this space with the standard skew-symmetric bilinear form.

3) Denote by $\ell_{m}$ the space $\mathbb{Q}_{p}^{m}$ equipped with the standard symmetric bilinear form

$$
(z, w)=\sum z_{j} w_{j}
$$

We regard $x_{j}^{ \pm}, y_{j}^{ \pm}$as elements of this space.

Consider the tensor product $\mathcal{H} \otimes_{\mathbb{Q}_{p}} \ell_{m}$, vectors

$$
\left(\begin{array}{llllll}
x_{1}^{+} & \ldots & x_{k}^{+} & x_{1}^{-} & \ldots & x_{k}^{-}
\end{array}\right),\left(\begin{array}{llllll}
y_{1}^{+} & \ldots & y_{k}^{+} & y_{1}^{-} & \ldots & y_{k}^{-}
\end{array}\right)
$$

are regarded as elements of $\mathcal{H} \otimes \ell_{m}$. We equip $\mathcal{H} \otimes \ell_{m}$ with the tensor product of bilinear forms, this form is a skew-symmetric with matrix 16

$$
\left(\begin{array}{cccccc}
0 & \ldots & 0 & 1_{m} & \ldots & 0 \\
\vdots & \ddots & \vdots & \vdots & \ddots & \vdots \\
0 & \ldots & 0 & 0 & \ldots & 1_{m} \\
-1_{m} & \ldots & 0 & 0 & \ldots & 0 \\
\vdots & \ddots & \vdots & \vdots & \ddots & \vdots \\
0 & \ldots & -1_{m} & 0 & \ldots & 0
\end{array}\right) .
$$

Thus the operator in (4.2) is an operator

$$
\mathcal{V} \oplus\left(\mathcal{H} \otimes \ell_{m}\right) \quad \rightarrow \quad \mathcal{V} \oplus\left(\mathcal{H} \otimes \ell_{m}\right)
$$

We equip the spaces $\mathcal{V} \oplus\left(\mathcal{H} \otimes \ell_{m}\right)$ with a skew-symmetric bilinear form that is a direct sum of forms in $\mathcal{V}$ and $\mathcal{H} \otimes \ell_{m}$. The matrix of this form is

$$
\left(\begin{array}{cccc}
0 & 1_{\alpha} & 0 & 0 \\
-1_{\alpha} & 0 & 0 & 0 \\
0 & 0 & 0 & 1_{k m} \\
0 & 0 & -1_{k m} & 0
\end{array}\right)
$$

Evidently, operators (4.2) preserve this form, i.e., they are contained in $\operatorname{Sp}(2(\alpha+$ $\left.k m), \mathbb{Q}_{p}\right)$.

Now we start a description of characteristic functions.

\footnotetext{
${ }^{16}$ A tensor product of a symmetric and a skew-symmetric bilinear forms is a skew-symmetric bilinear form.
} 
For any self-dual module $Q \subset \mathcal{H}$ we consider the self-dual module

$$
Q \otimes_{\mathbb{Z}_{p}} \mathbb{Z}_{p}^{m} \subset \mathcal{H} \otimes \ell_{m}
$$

Notice, that $Q \otimes \mathbb{Z}_{p}^{m}$ is a direct sum of $m$ copies of $Q$.

Definition 4.1 Fix $g$. Fix self-dual submodules $Q, T \subset \mathcal{H}$. We define a relation

$$
\chi_{g}(Q, T): \mathcal{V} \rightrightarrows \mathcal{V}
$$

as the set of all $u \oplus v \in \mathcal{V} \oplus \mathcal{V}$ for which there exist $x \in Q \otimes \mathbb{Z}_{p}^{m}, y \in T \otimes \mathbb{Z}_{p}^{m}$ such that (4.2) holds.

\subsection{An auxiliary definition.}

Definition 4.2 We say that some property of a double coset holds in a general position if for any sufficiently large $m$ the set of points $g \in \mathrm{GL}\left(\alpha+k m, \mathbb{Q}_{p}\right)$, where the property does not hold, is a proper algebraic subvariety in $\operatorname{GL}(\alpha+$ $\left.k m, \mathbb{Q}_{p}\right)$.

\subsection{Basic properties of characteristic functions.}

Lemma $4.3 \chi_{g}(Q, T)$ does not depend on a choice of $m$.

Theorem 4.4 If $g_{1}, g_{2}$ are contained in the same double coset $\mathbf{K} \backslash \mathbf{G} / \mathbf{K}$, then $\chi_{g_{1}}(Q, T)=\chi_{g_{2}}(Q, T)$.

Thus, for any double coset $\mathfrak{g} \in \mathbf{K} \backslash \mathbf{G} / \mathbf{K}$ we get a well-defined map

$$
\chi_{\mathfrak{g}}: \operatorname{LMod}(\mathcal{H}) \times \operatorname{LMod}(\mathcal{H}) \rightarrow\{\text { space of relations } \mathcal{V} \rightrightarrows \mathcal{V}\}
$$

Therefore, we can write

$$
\chi_{\mathfrak{g}}(Q, T), \quad \text { where } \mathfrak{g} \in \mathbf{K} \backslash \mathbf{G} / \mathbf{K} .
$$

We say that $\chi_{\mathfrak{g}}(\cdot, \cdot)$ is the characteristic function of the double coset $\mathfrak{g}$.

Theorem $4.5 \chi_{\mathfrak{g}}(Q, T) \in \overline{\operatorname{Naz}}(\mathcal{V}, \mathcal{V})$.

Theorem 4.6 The following identity holds

$$
\chi_{\mathfrak{g} \star \mathfrak{h}}(Q, T)=\chi_{\mathfrak{g}}(Q, T) \chi_{\mathfrak{h}}(Q, T),
$$

in the right-hand side we have a product of relations 
4.4. Refinement of Theorem 4.5. Fix a double coset $\mathfrak{g}$. Substituting $x^{ \pm}=0, y^{ \pm}=0$ to the equation (4.2), we get an equation for $u \oplus v \in \mathcal{V} \oplus \mathcal{V}$. The explicit form (see equation (5.3)) is

$$
\left\{\begin{array}{l}
v^{+}=a u^{+} \\
0=c_{j} u^{+}, \quad \text { for all } j \\
u^{-}=a^{t} v^{-} \\
0=b_{j}^{t} v^{-}, \quad \text { for all } j
\end{array}\right.
$$

Denote by $\Lambda(\mathfrak{g}) \subset \mathcal{V} \oplus \mathcal{V}$ the linear subspace of solutions of this system.

Notice that

$$
\operatorname{ker} \Lambda(\mathfrak{g})=0, \quad \operatorname{indef} \Lambda(\mathfrak{g})=0
$$

(since $g$ is an invertible matrix).

For $\mathfrak{g}$ being in a general position $\Lambda(\mathfrak{g})=0$.

Proposition 4.7 a) For any self-dual $Q, T \in \operatorname{LMod}(\mathcal{H})$,

$$
\chi_{\mathfrak{g}}(Q, T)_{\downarrow} \supset \Lambda(\mathfrak{g}), \quad \chi_{\mathfrak{g}}(Q, T)^{\uparrow} \subset \Lambda(\mathfrak{g})^{\perp} .
$$

b) If $Q, T$ are self-dual lattices, then

$$
\chi_{\mathfrak{g}}(Q, T)_{\downarrow}=\Lambda(\mathfrak{g}), \quad \chi_{\mathfrak{g}}(Q, T)^{\uparrow}=\Lambda(\mathfrak{g})^{\perp} .
$$

Corollary 4.8 For $\mathfrak{g}$ being in a general position, we get a map

$$
\operatorname{LLat}(\mathcal{H}) \times \operatorname{LLat}(\mathcal{H}) \rightarrow \operatorname{LLat}(\mathcal{V} \oplus \mathcal{V}) .
$$

\subsection{Values of characteristic functions on the distinguished bound-} ary.

Theorem 4.9 Let $Q, T$ range in the Lagrangian Grassmannian $\operatorname{LGr}(\mathcal{H})$. Then

a) $\chi_{\mathfrak{g}}(Q, T)$ is a Lagrangian subspace in $\mathcal{V} \oplus \mathcal{V}$.

b) The map

$$
\chi_{\mathfrak{g}}: \operatorname{LGr}(\mathcal{H}) \times \operatorname{LGr}(\mathcal{H}) \rightarrow \operatorname{LGr}(\mathcal{V} \oplus \mathcal{V})
$$

is rational.

c) For $\mathfrak{g}$ being in a general position, $\chi_{\mathfrak{g}}(Q, T) \in \operatorname{Sp}\left(\mathcal{V}, \mathbb{Q}_{p}\right)$ a.s. on $\operatorname{LGr}(\mathcal{H}) \times$ $\operatorname{LGr}(\mathcal{H})$.

A precise description of the subset of $\mathbf{K} \backslash \mathbf{G} / \mathbf{K}$, where the last property holds, is given below in Subsection [5.9.

There is a more exotic statement in the same spirit.

Proposition 4.10 For all $\mathfrak{g}$ for almost all $(Q, T) \in \operatorname{LGr}(\mathcal{H}) \times \operatorname{LGr}(\mathcal{H})$, the condition $\left(u^{+} \oplus u^{-}\right) \oplus\left(v^{+} \oplus v^{-}\right) \in \chi_{\mathfrak{g}}(Q, T)$ can be written as an equation

$$
\left(\begin{array}{c}
v^{+} \\
u^{-}
\end{array}\right)=Z(Q, T)\left(\begin{array}{l}
v^{-} \\
u^{+}
\end{array}\right)
$$

there $Z(Q, T)$ is a symmetric matrix. 


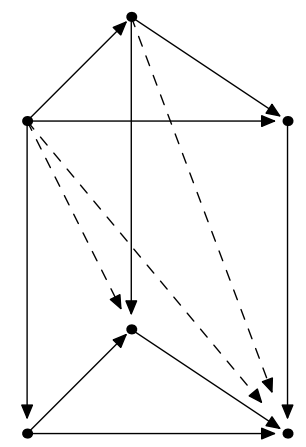

Figure 2: A reference to Subsection 4.6. A product of two simplices and additional arrows.

Point out that this can done for all $\mathfrak{g}$.

Proposition 4.11 Let

$$
\mathfrak{g}_{1}, \mathfrak{g}_{2} \in \mathbf{K} \backslash \mathbf{G} / \mathbf{K}=\mathrm{O}\left(\infty, \mathbb{Z}_{p}\right) \backslash \mathbf{G} / \mathrm{O}\left(\infty, \mathbb{Z}_{p}\right)
$$

be contained in the same double coset

$$
\mathrm{O}\left(\infty, \mathbb{Q}_{p}\right) \backslash \mathbf{G} / \mathrm{O}\left(\infty, \mathbb{Q}_{p}\right),
$$

then the restrictions of $\chi_{\mathfrak{g}_{1}}$ and $\chi_{\mathfrak{g}_{2}}$ to $\operatorname{LGr}(\mathcal{H}) \times \operatorname{LGr}(\mathcal{H})$ coincide.

4.6. Extension of characteristic function to buildings. Next, consider two almost self-dual submodules $Q, T$ and apply to them the definition of characteristic function $Q, T$.

Proposition 4.12 If $Q, T$ are almost self-dual modules, then $\chi_{\mathfrak{g}}(Q, T)$ is almost self-dual.

Now we construct an oriented graph $\Delta(\mathcal{H} \bowtie \mathcal{H})$. Vertices are ordered pairs $(Q, T)$ of almost self-dual submodules in $\mathcal{H}$. We draw an arrow from $(Q, T)$ to $\left(Q^{\prime}, T^{\prime}\right)$ if $Q \supset Q^{\prime}, T \supset T^{\prime}$.

Consider the product of simplicial complexes $\operatorname{Bd}(\mathcal{H}) \times \operatorname{Bd}(\mathcal{H})$. It is polyhedral complex, whose cells are products of simplices. Two vertices (of this complex) $(Q, T)$ and $\left(Q^{\prime}, T^{\prime}\right)$ are connected by an arrow if $Q \supset Q^{\prime}$ and $T=T^{\prime}$ or $Q=Q^{\prime}$ and $T \supset T^{\prime}$. However, our rule from the previous paragraph produces more arrows, this provides a simplicial partition of each product of simplices (see, e.g., 44, Section 3.B). Finally, we get a $2 k$-dimensional simplicial complex $\operatorname{Bd}(\mathcal{H} \bowtie \mathcal{H})$ (it also is a subcomplex of the complex $\operatorname{Bd}(\mathcal{H} \oplus \mathcal{H})$ ).

Let $\Phi, \Psi$ be two oriented graphs, assume that number of edges connecting any pair of vertices is $\leqslant 1$. We say that a map $\sigma: \operatorname{Vert}(\Phi) \rightarrow \operatorname{Vert}(\Psi)$ is a morphism of graphs if for any arrow $a \rightarrow b$ in $\Phi$ we have $\sigma(a)=\sigma(b)$ or there is an arrow $\sigma(a) \rightarrow \sigma(b)$. 


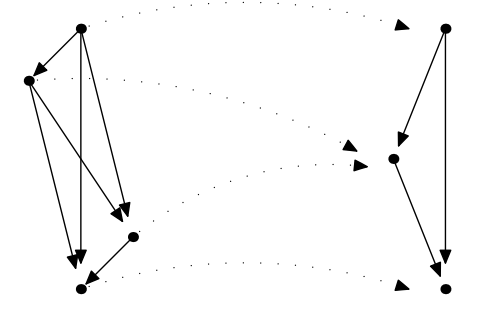

Figure 3: A reference to Subsection 4.6. A morphism of oriented graphs

Theorem 4.13 A characteristic function $\chi_{\mathfrak{g}}$ is a morphism of oriented graphs

$$
\Delta(\mathcal{H} \bowtie \mathcal{H}) \rightarrow \Delta(\mathcal{V} \oplus \mathcal{V})
$$

\subsection{Continuity.}

Theorem 4.14 Let $Q_{j}, Q, T_{j}, T$ be almost self-dual modules. If $Q_{j} \nearrow Q$, $T_{j} \nearrow T$, then

$$
\chi_{\mathfrak{g}}\left(Q_{j}, T_{j}\right) \nearrow \chi_{\mathfrak{g}}(Q, T)
$$

Notice that characteristic function can be discontinuous with respect to the Hausdorff convergence. Moreover, the restriction of $\chi_{\mathfrak{g}}$ to $\operatorname{LGr}(\mathcal{H}) \times \operatorname{LGr}(\mathcal{H})$ can be discontinuous in the topology of Grassmannian.

\subsection{Involution.}

Proposition 4.15 If $u \oplus v \in \chi_{\mathfrak{g}}(Q, T)$, then $v \oplus u \in \chi_{\mathfrak{g}^{*}}(T, Q)$.

4.9. Additional symmetry. For a nonzero $\lambda \in \mathbb{Q}_{p}^{\times}=\mathbb{Q}_{p}$, we define an operator $M(\lambda)$ in $\mathcal{H}$ given by $\left(\begin{array}{cc}\lambda & 0 \\ 0 & \lambda^{-1}\end{array}\right)$, by the same symbol we denote the operator $\left(\begin{array}{cc}\lambda & 0 \\ 0 & \lambda^{-1}\end{array}\right)$ in the space $\mathcal{V}$.

\section{Theorem 4.16}

$$
\chi_{\mathfrak{g}}(M(\lambda) Q, M(\lambda) T)=M\left(\lambda^{-1}\right) \chi_{\mathfrak{g}}(Q, T) M(\lambda)
$$

4.10. Remark. Another semigroup of double cosets. Consider the group $\widetilde{\mathbf{G}}=\operatorname{Sp}\left(2 \alpha+2 k \infty, \mathbb{Q}_{p}\right)$ of symplectic matrices $\left(\begin{array}{ll}a & b \\ c & d\end{array}\right)$ of size $(\alpha+k \infty)+$ $(\alpha+k \infty), \widetilde{\mathbf{G}} \supset \mathbf{G}$. Consider its subgroup $\mathbf{G}=\mathrm{GL}\left(\alpha+k \infty, \mathbb{Q}_{p}\right)$ consisting of matrices $\left(\begin{array}{cc}g & 0 \\ 0 & g^{t-1}\end{array}\right)$, consider the same $\mathbf{K}=\mathrm{O}\left(\infty, \mathbb{Z}_{p}\right) \subset \mathrm{GL}\left(\alpha+k \infty, \mathbb{Q}_{p}\right)$. Consider the semigroup of double cosets $\mathbf{K} \backslash \widetilde{\mathbf{G}} / \mathbf{K}$, the multiplication is determined as in Theorem 2.1. 
We define characteristic function $\chi_{\widetilde{\mathfrak{g}}}(Q, T)$ in the same way, in formula (4.2) instead the matrix $\left(\begin{array}{cc}g & 0 \\ 0 & g^{t-1}\end{array}\right)$ we write a symplectic matrix $\left(\begin{array}{ll}a & b \\ c & d\end{array}\right) \in \operatorname{Sp}(2 \alpha+$ $\left.2 k \infty, \mathbb{Q}_{p}\right)$.

Theorem 4.17 All the statements of this section hold for $\chi_{\mathfrak{\mathfrak { g }}}(Q, T)$ except Theorem 4.16 and Proposition 4.1017.

\section{Proofs}

5.1. Independence of representatives. To shorten expressions, set $k=2$. Let $h \in \mathrm{O}\left(m, \mathbb{Z}_{p}\right)$, let $\mathfrak{I}(h)$ be given by (2.2). Then characteristic function of $g \Im(h)$ is determined by

$$
\left(\begin{array}{l}
v^{+} \\
y_{1}^{+} \\
y_{2}^{+} \\
v^{-} \\
y_{1}^{-} \\
y_{2}^{-}
\end{array}\right)=\left(\begin{array}{cclrll}
a & b_{1} h & b_{2} h & 0 & 0 & 0 \\
c_{1} & d_{11} h & d_{12} h & 0 & 0 & 0 \\
c_{2} & d_{21} h & d_{22} h & 0 & 0 & 0 \\
0 & 0 & 0 \\
0 & 0 & 0 \\
0 & 0 & 0
\end{array} \quad\left(\begin{array}{ccc}
a & b_{1} h & b_{2} h \\
c_{1} & d_{11} h & d_{12} h \\
c_{2} & d_{21} h & d_{22} h
\end{array}\right)^{t-1}\right)\left(\begin{array}{l}
u^{+} \\
x_{1}^{+} \\
x_{2}^{+} \\
u^{-} \\
x_{1}^{-} \\
x_{2}^{-}
\end{array}\right) .
$$

or

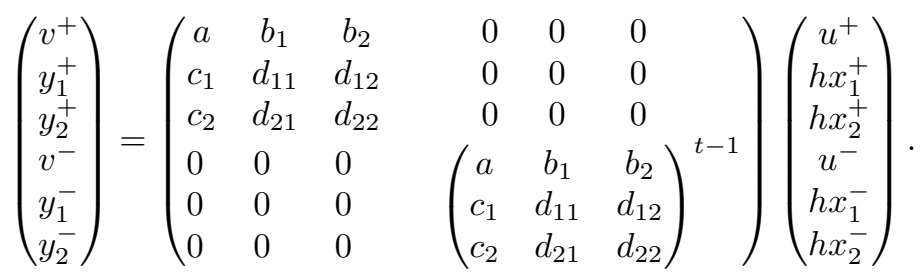

We introduce new variables $\widetilde{x}_{1}^{ \pm}=h x_{1}^{ \pm}, \widetilde{x}_{2}^{ \pm}=h x_{2}^{ \pm}$and come to the equation for $\chi_{g}$. Notice that modules $Q \otimes_{\mathbb{Z}_{p}} \mathbb{Z}_{p}^{m}$ are invariant with respect to $\mathrm{O}\left(m, \mathbb{Z}_{p}\right)$.

5.2. Proof of Proposition 4.11. Proof is the same, we only take $h \in$ $\mathrm{O}\left(m, \mathbb{Q}_{p}\right)$. If $Q \subset \mathcal{H}$ is a subspace, then $Q \otimes \ell_{m}=Q \otimes \mathbb{Q}_{p}^{m}$ is a subspace, it is $\mathrm{O}\left(m, \mathbb{Q}_{p}\right)$-invariant.

5.3. Reformulation of definition. The equation (4.2) determines a linear subspace in

$$
\left(\mathcal{V} \oplus\left(\mathcal{H} \otimes \ell_{m}\right)\right) \oplus\left(\mathcal{V} \oplus\left(\mathcal{H} \otimes \ell_{m}\right)\right) .
$$

We regard it as a linear relation

$$
\xi:\left(\left(\mathcal{H} \otimes \ell_{m}\right) \oplus\left(\mathcal{H} \otimes \ell_{m}\right)\right) \rightrightarrows(\mathcal{V} \oplus \mathcal{V})
$$

Then $\chi_{\mathfrak{g}}$ is the image of the submodule

$$
\eta_{Q, T}=\left(Q \otimes_{\mathbb{Z}_{p}} \mathbb{Z}_{p}^{m}\right) \oplus\left(T \otimes_{\mathbb{Z}_{p}} \mathbb{Z}_{p}^{m}\right)
$$

\footnotetext{
${ }^{17}$ the system (4.4) also must be modified.
} 
under $\xi$.

5.4. Immediate corollaries. The relation $\xi$ is a morphism of the category

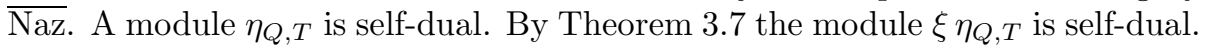
Theorem 4.5 is proved.

The same argument implies Theorem 4.9]a and Proposition 4.12,

Also Lemma 4.3 became obvious.

5.5. Continuity (Theorem 4.14). We refer to Theorem 3.11,

5.6. Products. Proof of Theorem 4.6. To shorten notation, set $k=2$. Let

$g=\left(\begin{array}{ccc}a & b_{1} & b_{2} \\ c_{1} & d_{11} & d_{12} \\ c_{2} & d_{21} & d_{22}\end{array}\right) \in \mathrm{GL}\left(\alpha+2 l, \mathbb{Q}_{p}\right), \quad h=\left(\begin{array}{ccc}a^{\prime} & b_{1}^{\prime} & b_{2}^{\prime} \\ c_{1}^{\prime} & d_{11}^{\prime} & d_{12}^{\prime} \\ c_{2}^{\prime} & d_{21}^{\prime} & d_{22}^{\prime}\end{array}\right) \in \mathrm{GL}\left(\alpha+2 m, \mathbb{Q}_{p}\right)$.

Let $v \oplus w \in \chi_{\mathfrak{g}}(Q, T), u \oplus v \in \chi_{\mathfrak{h}}(Q, T)$. Then there are $x \in Q \otimes_{\mathbb{Z}_{p}} \mathbb{Z}_{p}^{m}$, $y \in T \otimes_{\mathbb{Z}_{p}} \mathbb{Z}_{p}^{m}$ such that

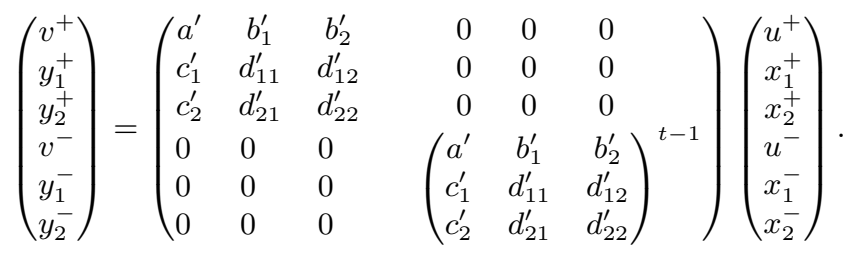

Also there are $X \in Q \otimes_{\mathbb{Z}_{p}} \mathbb{Z}_{p}^{l}, Y \in T \otimes_{\mathbb{Z}_{p}} \mathbb{Z}_{p}^{l}$ such that

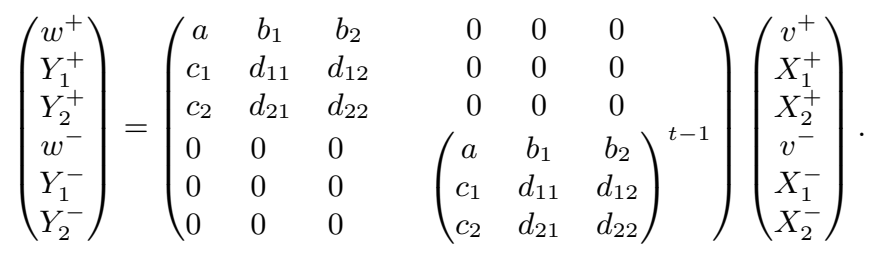

We write (5.2) as

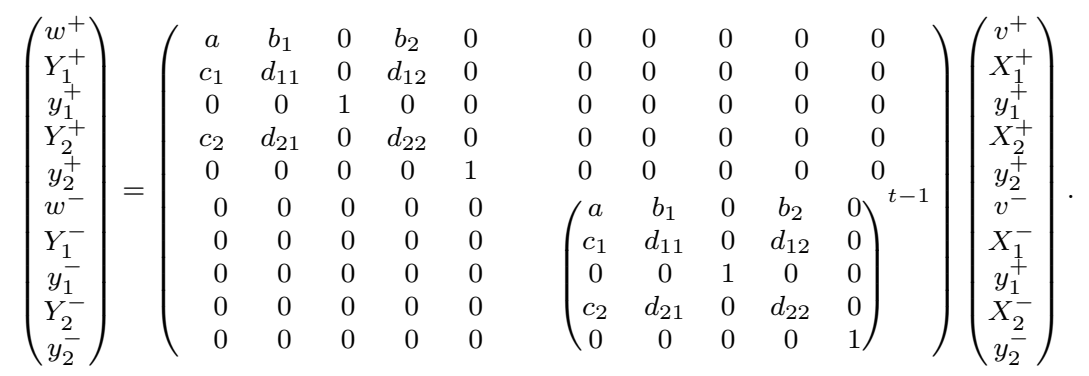


Applying (5.1) we come to

$$
\begin{aligned}
& \left(\begin{array}{c}
w^{+} \\
Y_{1}^{+} \\
y_{1}^{+} \\
Y_{2}^{+} \\
y_{2}^{+} \\
w^{-} \\
Y_{1}^{-} \\
y_{1}^{-} \\
Y_{2}^{-} \\
y_{2}^{-}
\end{array}\right)=\left(\begin{array}{cccccccccc}
a & b_{1} & 0 & b_{2} & 0 & 0 & 0 & 0 & 0 & 0 \\
c_{1} & d_{11} & 0 & d_{12} & 0 & 0 & 0 & 0 & 0 & 0 \\
0 & 0 & 1 & 0 & 0 & 0 & 0 & 0 & 0 & 0 \\
c_{2} & d_{21} & 0 & d_{22} & 0 & 0 & 0 & 0 & 0 & 0 \\
0 & 0 & 0 & 0 & 1 & 0 & 0 & 0 & 0 & 0 \\
0 & 0 & 0 & 0 & 0 \\
0 & 0 & 0 & 0 & 0 \\
0 & 0 & 0 & 0 & 0 \\
0 & 0 & 0 & 0 & 0 \\
0 & 0 & 0 & 0 & 0 & \left(\begin{array}{ccccc}
a & b_{1} & 0 & b_{2} & 0 \\
c_{1} & d_{11} & 0 & d_{12} & 0 \\
0 & 0 & 1 & 0 & 0 \\
c_{2} & d_{21} & 0 & d_{22} & 0 \\
0 & 0 & 0 & 0 & 1
\end{array}\right)^{t-1}
\end{array}\right) \times
\end{aligned}
$$

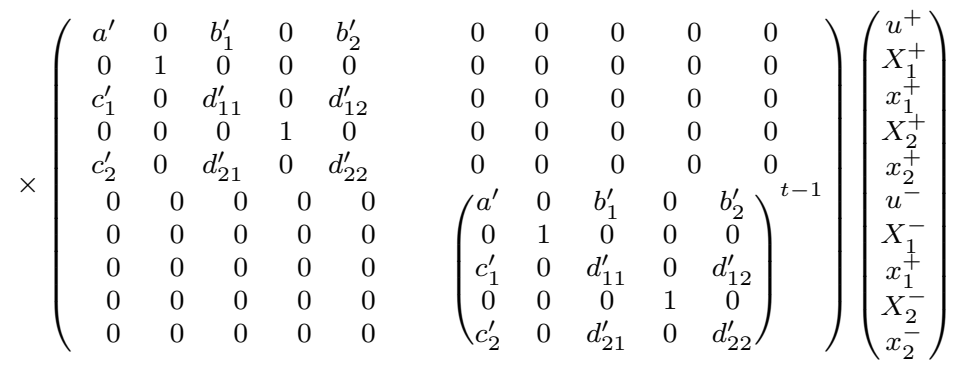

Now

$$
X \oplus x \in Q \otimes\left(\mathbb{Z}_{p}^{l} \oplus \mathbb{Z}_{p}^{m}\right), \quad Y \oplus y \in T \otimes\left(\mathbb{Z}_{p}^{l} \oplus \mathbb{Z}_{p}^{m}\right),
$$

and we get $u \oplus w \in \chi_{\mathfrak{g} \star \mathfrak{h}}(Q, T)$. Thus,

$$
\chi_{\mathfrak{g} \star \mathfrak{h}}(Q, T) \supset \chi_{\mathfrak{g}}(Q, T) \chi_{\mathfrak{h}}(Q, T) .
$$

But both sides are self-dual, therefore they coincide.

5.7. Morphisms of graphs (Theorem 4.13). Consider the map

$$
\operatorname{LMod}(\mathcal{H}) \times \operatorname{LMod}(\mathcal{H}) \rightarrow \operatorname{LMod}\left(\mathcal{H} \otimes \ell_{m}\right) \times \operatorname{LMod}\left(\mathcal{H} \otimes \ell_{m}\right)
$$

given by $(Q, T) \mapsto\left(Q \otimes_{\mathbb{Z}_{p}} \mathbb{Z}_{p}^{m}, T \otimes_{\mathbb{Z}_{p}} \mathbb{Z}_{p}^{m}\right)$.

Lemma 5.1 This map is a morphism of graphs

$$
\Delta(\mathcal{H} \bowtie \mathcal{H}) \rightarrow \Delta\left(\left(\mathcal{H} \otimes \ell_{m}\right) \bowtie\left(\mathcal{H} \otimes \ell_{m}\right)\right) .
$$

This statement is obvious.

Next, we have an embedding of complexes

$$
\operatorname{Bd}\left(\left(\mathcal{H} \otimes \ell_{m}\right) \bowtie\left(\mathcal{H} \otimes \ell_{m}\right)\right) \rightarrow \operatorname{Bd}\left(\left(\mathcal{H} \otimes \ell_{m}\right) \oplus\left(\mathcal{H} \otimes \ell_{m}\right)\right) .
$$

On the other hand, the linear relation $\xi$ is a morphism of the category Naz. Therefore it induces a morphism of graphs $\Delta\left(\left(\mathcal{H} \otimes \ell_{m}\right) \oplus\left(\mathcal{H} \otimes \ell_{m}\right)\right) \rightarrow \Delta(\mathcal{V} \oplus \mathcal{V})$, see 9], Proposition 10.7.6.

5.8. Proof of Proposition 4.7. We have

$$
\text { indef } \xi=\Lambda(\mathfrak{g}) \text {. }
$$


Therefore $\Lambda(\mathfrak{g}) \subset \xi \eta_{Q, T} \subset \Lambda(\mathfrak{g})^{\perp}$. This is the statement a) of Proposition 4.7.

Also, if $R$ is a relation $\mathcal{V} \rightrightarrows W, Y \subset \mathcal{V}$ is a lattice, then $(R Y)_{\downarrow}=(\text { indef } R)_{\downarrow}$. This implies b).

5.9. Values on the distinguished boundary. Now let $Q, T$ be Lagrangian subspaces in $\mathcal{H}$.

Proof of Proposition 4.10, Decompose $\mathcal{H}=\mathcal{H}_{+} \oplus \mathcal{H}_{-}=\mathbb{Q}_{p}^{\alpha} \oplus \mathbb{Q}_{p}^{\alpha}$. A Lagrangian subspace $Q \subset \mathcal{H}$ of general position is a graph of an operator $\mathcal{H}^{+} \rightarrow \mathcal{H}^{-}$, and matrix of this operator is symmetric (see, e.g., [9], Theorem 3.1.4). To shorten notation, set $k=2$. The equation (4.2) can be written in the form

$$
\left(\begin{array}{c}
v^{+} \\
y_{1}^{+} \\
y_{2}^{+} \\
u^{-} \\
t_{11} x_{1}^{+}+t_{12} x_{2}^{+} \\
t_{12} x_{1}^{+}+t_{22} x_{2}^{+}
\end{array}\right)\left(\begin{array}{cccccc}
a & b_{1} & b_{2} & 0 & 0 & 0 \\
c_{1} & d_{11} & d_{12} & 0 & 0 & 0 \\
c_{2} & d_{21} & d_{22} & 0 & 0 & 0 \\
0 & 0 & 0 & a^{t} & c_{1}^{t} & c_{2}^{t} \\
0 & 0 & 0 & b_{1}^{t} & d_{11}^{t} & d_{21}^{t} \\
0 & 0 & 0 & b_{2}^{t} & d_{12}^{t} & d_{22}^{t}
\end{array}\right)\left(\begin{array}{c}
u^{+} \\
x_{1}^{+} \\
x_{2}^{+} \\
v^{-} \\
q_{11} y_{1}^{+}+q_{12} y_{2}^{+} \\
q_{12} y_{1}^{+}+q_{22} y_{2}^{+}
\end{array}\right),
$$

We denote

$$
\varkappa:=\left(\begin{array}{ll}
q_{11} & q_{12} \\
q_{12} & q_{22}
\end{array}\right), \quad \tau:=\left(\begin{array}{cc}
t_{11} & t_{12} \\
t_{12} & t_{22}
\end{array}\right)
$$

and write (5.3) as

$$
\begin{aligned}
& v^{+}=a u^{+}+b x^{+} \\
& y^{+}=c u^{+}+d x^{+} \\
& u^{-}=a^{t} v^{-}+c^{t} \varkappa y^{+} \\
& \tau x^{+}=b^{t} v^{-}+d^{t} \varkappa y^{+} .
\end{aligned}
$$

We regard lines (5.5), (5.7) as a system of equations for $x^{+}, y^{+}$. The matrix of the system is

$$
\Omega(\varkappa, \tau)=\left(\begin{array}{cc}
-d & 1 \\
\tau & -d^{t} \varkappa
\end{array}\right) .
$$

Evidently, the polynomial $\operatorname{det} \Omega(\varkappa, \tau)$ is not zero. Indeed, fix $\varkappa$ and take $\tau=p^{-N}$. 1. If $N$ is sufficiently large, then the determinant is $\neq 0$. Thus, outside the hypersurface

$$
\operatorname{det} \Omega(\varkappa, \tau)=0
$$

we can express $x^{+}$and $y^{+}$as functions of $u^{+}, v^{-}$. After substitution of $x^{+}, y^{+}$ to (5.4), (5.6), we get a dependence of $u^{-}, v^{+}$in $u^{+}, v^{-}$.

This also proves Theorem 4.9, b (rationality of characteristic function).

Proof Theorem 4.9, c. Denote

$$
\left(\begin{array}{ll}
a & b \\
c & d
\end{array}\right)^{-1}=\left(\begin{array}{ll}
A & B \\
C & D
\end{array}\right)
$$


and write the equation (4.2) in the form

$$
\left(\begin{array}{c}
v^{+} \\
y_{1}^{+} \\
y_{2}^{+} \\
v^{-} \\
q_{11} y_{1}^{+}+q_{12} y_{2}^{+} \\
q_{12} y_{1}^{+}+q_{22} y_{2}^{+}
\end{array}\right)\left(\begin{array}{cccccc}
a & b_{1} & b_{2} & 0 & 0 & 0 \\
c_{1} & d_{11} & d_{12} & 0 & 0 & 0 \\
c_{2} & d_{21} & d_{22} & 0 & 0 & 0 \\
0 & 0 & 0 & A^{t} & C_{1}^{t} & C_{2}^{t} \\
0 & 0 & 0 & B_{1}^{t} & D_{11}^{t} & D_{21}^{t} \\
0 & 0 & 0 & B_{2}^{t} & D_{12}^{t} & D_{22}^{t}
\end{array}\right)\left(\begin{array}{c}
u^{+} \\
x_{1}^{+} \\
x_{2}^{+} \\
u^{-} \\
t_{11} x_{1}^{+}+t_{12} x_{2}^{+} \\
t_{12} x_{1}^{+}+t_{22} x_{2}^{+}
\end{array}\right)
$$

or

$$
\begin{array}{r}
v^{+}=a u^{+}+b x^{+} \\
y^{+}=c u^{+}+d x^{+} \\
v^{-}=A^{t} u^{-}+C^{t} \tau x^{+} \\
y_{+}=B^{t} u^{-}+D^{t} \tau x^{+} .
\end{array}
$$

We consider lines (5.9), (5.11) as equations for $y^{+}, x^{+}$. The matrix of the system is

$$
\Xi(\varkappa, \tau)=\left(\begin{array}{cc}
1 & -d \\
\varkappa & -D^{t} \tau
\end{array}\right) .
$$

Its determinant equals

$$
\operatorname{det} \Xi(\varkappa, \tau)=\operatorname{det}\left(-D^{t} \tau+\varkappa d\right)
$$

If it is nonzero, we get a linear operator $u \mapsto v$. We come to the following statement:

Proposition 5.2 If there exists a pair of symmetric matrices $\varkappa$, $\tau$ such that $\operatorname{det}\left(-D^{t} \tau+\varkappa d\right) \neq 0$, then $\chi_{\mathfrak{g}}(Q, T) \in \operatorname{Sp}\left(\mathcal{V}, \mathbb{Q}_{p}\right)$ a.s. on $\operatorname{LGr}(\mathcal{H}) \times \operatorname{LGr}(\mathcal{H})$.

5.10. Involution. Proof of Proposition 4.15. We write the defining relation for $\chi_{g^{-1}}$

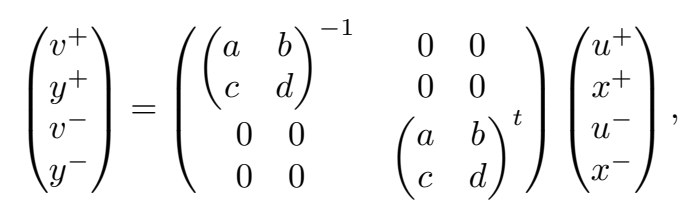

represent this in the form

$$
\left(\begin{array}{l}
u^{+} \\
x^{+} \\
u^{-} \\
x^{-}
\end{array}\right)=\left(\begin{array}{llll}
a & b & 0 & 0 \\
c & d & 0 & 0 \\
0 & 0 & \left(\begin{array}{ll}
a & b \\
c & d
\end{array}\right)^{t-1}
\end{array}\right)\left(\begin{array}{l}
v^{+} \\
y^{+} \\
v^{-} \\
y^{-}
\end{array}\right)
$$

and come to desired statement. 
5.11. Proof of Theorem 4.16. We write (4.2) as

$$
\left(\begin{array}{l}
u^{+} \\
x^{+} \\
u^{-} \\
x^{-}
\end{array}\right)=\left(\begin{array}{cccc}
\lambda^{-1} & & & \\
& \lambda^{-1} & & \\
& & \lambda & \\
& & & \lambda
\end{array}\right)\left(\begin{array}{cccc}
a & b & 0 & 0 \\
c & d & 0 & 0 \\
0 & 0 & \left(\begin{array}{ll}
a & b \\
c & d
\end{array}\right)
\end{array}\right)\left(\begin{array}{cccc}
\lambda & & & \\
& \lambda & & \\
& & \lambda^{-1} & \\
& & & \lambda^{-1}
\end{array}\right)\left(\begin{array}{l}
v^{+} \\
y^{+} \\
v^{-} \\
y^{-}
\end{array}\right)
$$

or

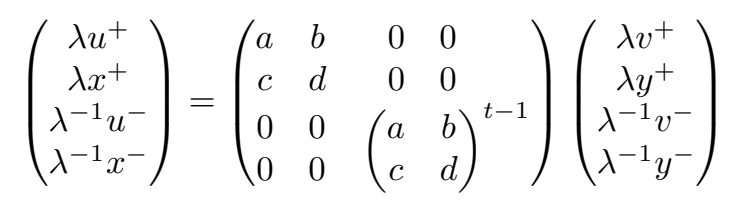

5.12. Another reformulation of the definition of characteristic functions. Consider the space $W=\mathcal{V} \oplus\left(\mathcal{H} \otimes \ell_{m}\right)$. For any self-dual submodule $Q \subset \mathcal{H}$, consider the linear relation $\Lambda: \mathcal{V} \rightrightarrows W$ defined by

$$
\Lambda_{Q}=1_{\mathcal{V}} \oplus\left(Q \otimes \mathbb{Z}_{p}^{m}\right) \subset(\mathcal{V} \oplus \mathcal{V}) \oplus\left(Q \otimes \ell_{m}\right) .
$$

Then $\chi_{\mathfrak{g}}$ is a product of linear relations

$$
\chi_{\mathfrak{g}}(Q, T)=\left(\Lambda_{T}\right)^{\square}\left(\begin{array}{cc}
g & 0 \\
0 & g^{t-1}
\end{array}\right) \Lambda_{Q} .
$$

\section{Multiplicativity theorem}

Theorem 2.2 (multiplicativity theorem) formulated above is a representative of wide class of theorems, their proofs are standard, below we refer to proofs [8], Chapter VIII.

\subsection{Corners of orthogonal matrices.}

Lemma 6.1 Let $A$ be a $m \times m$ matrix with elements $\in \mathbb{Z}_{p}$. Then there exists $N$ and a matrix $\left(\begin{array}{ll}A & B \\ C & D\end{array}\right) \in \mathrm{O}\left(m+N, \mathbb{Z}_{p}\right)$.

Proof. Denote by $\mathbf{B}_{m}$ the set of all possible $m \times m$ left upper corners of matrices $g \in \mathrm{O}\left(\infty, \mathbb{Z}_{p}\right)$.

1) The set $\mathbf{B}_{m}$ is closed with respect to matrix products. Indeed, let

$$
\left(\begin{array}{ll}
A & B \\
C & D
\end{array}\right) \in \mathrm{O}\left(m+N, \mathbb{Z}_{p}\right), \quad\left(\begin{array}{ll}
A^{\prime} & B^{\prime} \\
C^{\prime} & D^{\prime}
\end{array}\right) \in \mathrm{O}\left(m+N^{\prime}, \mathbb{Z}_{p}\right) .
$$

Then

$$
\begin{aligned}
& \left(\begin{array}{ccc}
A & B & 0 \\
C & D & 0 \\
0 & 0 & 1
\end{array}\right)\left(\begin{array}{ccc}
A^{\prime} & 0 & B^{\prime} \\
0 & 1 & 0 \\
C^{\prime} & 0 & D^{\prime}
\end{array}\right)=\left(\begin{array}{ccc}
A A^{\prime} & \ldots & \ldots \\
\ldots & \ldots & \cdots \\
\cdots & \ldots & \ldots
\end{array}\right) \in \mathrm{O}\left(m+N+N^{\prime}, \mathbb{Z}_{p}\right) \\
& \text { 2) If } A \in \mathbf{B}_{m}, A^{\prime} \in \mathbf{B}_{n}, \text { then }\left(\begin{array}{cc}
A & 0 \\
0 & A^{\prime}
\end{array}\right) \in \mathbf{B}_{m+n} .
\end{aligned}
$$


3) It is more-or-less clear that for any $z \in \mathbb{Z}_{p}$ we have

$$
(z) \in \mathbf{B}_{1}, \quad\left(\begin{array}{cc}
1 & z \\
0 & 1
\end{array}\right),\left(\begin{array}{cc}
1 & 0 \\
z & 1
\end{array}\right) \in \mathbf{B}_{2} .
$$

4) $\mathbf{B}_{m}$ contains matrices of permutations.

Now we can produce any matrix with integer elements.

6.2. Admissible representations. Denote by $\mathbf{K}_{m}$ the subgroup in $\mathbf{K}$ consisting of matrices of the form $\left(\begin{array}{cc}1_{m} & 0 \\ 0 & *\end{array}\right)$.

Let $\tau$ be a unitary representation of $\mathbf{K}$ in a Hilbert space $H$. Denote by $H(m)$ the subspace of $\mathbf{K}_{m}$-fixed vectors. Denote by $P(m)$ the operator of orthogonal projection to $H(m)$. We say, that $\tau$ is admissible if $\cup_{m} H(m)$ is dense in $H$.

We say, that a representation of $\mathbf{G}$ is $\mathbf{K}$-admissible if its restriction to $\mathbf{K}$ is admissible.

6.3. Continuation of representations. Denote by $\mathbf{B}_{\infty}$ the semigroup of all infinite matrices $A$ such that:

a) $a_{i j} \in \mathbb{Z}_{p}$;

b) for each $i$ the sequence $a_{i j}$ tends to 0 as $j \rightarrow \infty$; for each $j$ the sequence $a_{i j}$ tends to 0 as $i \rightarrow \infty$.

We say that a sequence of matrices $A^{(j)} \in \mathbf{B}_{\infty}$ weakly converges to $A$ if we have convergence of each matrix element, $a_{k l}^{(j)} \rightarrow a_{k l}$.

Denote by $\mathbf{O}\left(\infty, \mathbb{Z}_{p}\right)$ the group of all orthogonal matrices $\in \mathbf{B}_{\infty}$.

Lemma 6.2 The group $\mathrm{O}\left(\infty, \mathbb{Z}_{p}\right)$ is dense in $\mathbf{O}\left(\infty, \mathbb{Z}_{p}\right)$ and in $\mathbf{B}_{\infty}$.

Proof. Let $S \in \mathbf{B}_{\infty}$. Consider its left upper corner of size $m \times m$. Consider $g_{m} \in \mathbb{O}\left(\infty, \mathbb{Z}_{p}\right)$ having the same left upper corner. Then $g_{m}$ weakly converges to $S$,

Theorem 6.3 a) Let $\tau$ be a unitary representation of $\mathbf{K}=\mathrm{O}\left(\infty, \mathbb{Z}_{p}\right)$. The following conditions are equivalent:

- $\tau$ is admissible;

- $\tau$ admits a weakly continuous extension to the group $\mathbf{O}\left(\infty, \mathbb{Z}_{p}\right)$;

- $\tau$ admits a weakly continuous extension to a representation $\widetilde{\tau}$ of the semigroup $\mathbf{B}_{\infty}$ such that $\widetilde{\tau}\left(A^{t}\right)=\widetilde{\tau}(A)^{*},\|\widetilde{\tau}(A)\| \leqslant 1$ for all $A$.

b) For an admissible representation $\tau$,

$$
P(m)=\widetilde{\tau}\left(\begin{array}{cc}
1_{m} & 0 \\
0 & 0
\end{array}\right) .
$$

This is a statement in the spirit of [24. We omit a proof, since it is a one-to-one repetition of proof of [8], Theorem VIII.1.4 about symmetric groups (admissibility implies semigroup continuation), the only new detail is Lemma 6.1). Admissibility follows from continuity by [8, Proposition VIII.1.3. 
Corollary 6.4 Denote

$$
\Theta_{N}^{(m)}=\left(\begin{array}{cccc}
1_{m} & 0 & 0 & 0 \\
0 & 0 & 1_{N} & 0 \\
0 & 1_{N} & 0 & 0 \\
0 & 0 & 0 & 1_{\infty}
\end{array}\right) .
$$

The projector $P(m)$ is a weak limit of the sequence

$$
P(m)=\lim _{N \rightarrow \infty} \tau\left(\Theta_{N}^{(m)}\right) .
$$

Proof. The sequence $\Theta_{N}^{(m)} \in \mathrm{O}\left(\infty, \mathbb{Z}_{p}\right)$ weakly converges to the matrix $\left(\begin{array}{cc}1_{m} & 0 \\ 0 & 0\end{array}\right) \in \mathbf{B}_{\infty}$. We refer to the statement b) of the theorem.

6.4. Proof of Theorem 2.2, We keep the notation of Subsection 2.3, Let $v \in H^{\mathbf{K}}, g \in G_{j}=\mathrm{GL}\left(\alpha+k m, \mathbb{Q}_{p}\right)$, let $q \in \mathbf{K}_{j}$. Then

$$
\rho(q) \rho(g) v=\rho(g) \rho(q) h=\rho(g) h,
$$

i.e., $v \in H(j)$. Thus the subspace $\cup_{j} H(j)$ is $\mathbf{G}$-invariant. Its closure is an admissible representation of G. In $\left(\cup_{j} H(j)\right)^{\perp}$ Theorem 2.2 holds by a trivial reason (the space of fixed vectors $\mathbf{K}$ is zero).

Thus, without loss of generality we can assume that $\rho$ is admissible.

Now let $g, h \in \mathbf{G}$, let $\mathfrak{g}, \mathfrak{h} \in \mathbf{K} \backslash \mathbf{G} / \mathbf{K}$ be the corresponding double cosets. Let $P=P(0)$ be the projector to $\mathbf{K}$-fixed vectors. Applying Corollary 6.4, we obtain

$$
\bar{\rho}(\mathfrak{g}) \bar{\rho}(h)=P \rho(g) P \rho(h)=\lim _{N \rightarrow \infty} P \rho(g) \rho\left(\mathfrak{I}\left(\Theta_{N}^{(0)}\right)\right) \rho(h)=\lim _{N \rightarrow \infty} P \rho\left(g \mathfrak{I}\left(\Theta_{N}\right) h\right),
$$

here $\mathfrak{J}: \mathbf{K} \rightarrow \mathbf{G}$ is the embedding (2.2). By the definition $\left(\Theta_{N}^{(0)}\right.$ is $\Theta_{N}$ from Subsection 2.3), we get $\bar{\rho}(\mathfrak{g} \star \mathfrak{h})$.

6.5. Variation of construction. Train. We can define multiplication of double cosets

$$
\mathbf{K}_{p} \backslash \mathbf{G} / \mathbf{K}_{q} \times \mathbf{K}_{q} \backslash \mathbf{G} / \mathbf{K}_{r} \rightarrow \mathbf{K}_{p} \backslash \mathbf{G} / \mathbf{K}_{r} .
$$

In the definition of product of double cosets (Subsection 2.2), we simply change $\Theta_{N}$ by $\Theta_{N}^{(q)}$. An explicit formula of the product is the same (2.4). Thus we get a category (train $\mathcal{T}(\mathbf{G}, \mathbf{K})$ of the pair $(\mathbf{G}, \mathbf{K})$ ).

Next, for any unitary representation $\rho$ of the group $\mathbf{G}$, a double coset $\mathfrak{g} \in$ $\mathbf{K}_{p} \backslash \mathbf{G} / \mathbf{K}_{q}$ determines an operator $\bar{\rho}(\mathfrak{g}): H(q) \rightarrow H(p)$ by the formula

$$
\bar{\rho}(g):=P(q) \rho(g), \quad g \in \mathfrak{g} .
$$

For any

$$
\mathfrak{g} \in \mathbf{K}_{p} \backslash \mathbf{G} / \mathbf{K}_{q} \quad \mathfrak{h} \in \mathbf{K}_{q} \backslash \mathbf{G} / \mathbf{K}_{r}
$$


the following identity holds

$$
\rho(\mathfrak{g}) \rho(\mathfrak{h})=\rho(\mathfrak{g} \star \mathfrak{h}),
$$

i.e., we get a representation of the category $\mathcal{T}(\mathbf{G}, \mathbf{K})$. Also,

$$
\rho\left(\mathfrak{g}^{*}\right)=\rho(\mathfrak{g})^{*}, \quad\|\rho(\mathfrak{g})\| \leqslant 1 .
$$

Also it can be shown that

Theorem 6.5 This construction is a bijection between the set of $\mathbf{K}$-admissible unitary representations of $\mathbf{G}$ and the set of representations of the category $\mathcal{T}(\mathbf{G}, \mathbf{K})$ satisfying (6.2).

We omit a proof, since it is the same as in [16.

Also the construction of characteristic functions and their properties survive for double cosets $\mathbf{K}_{p} \backslash \mathbf{G} / \mathbf{K}_{q}$.

\section{Representations of the group G}

\subsection{Existence of representations. Let}

$$
\left(\begin{array}{cccc}
a & b_{1} & \ldots & b_{k} \\
c_{1} & d_{11} & \ldots & d_{1 k} \\
\vdots & \vdots & \ddots & \vdots \\
c_{k} & d_{k 1} & \ldots & d_{k k}
\end{array}\right) \in \mathrm{GL}\left(\alpha+k \infty, \mathbb{Q}_{p}\right) .
$$

Consider embedding $\mathrm{GL}\left(\alpha+k \infty, \mathbb{Q}_{p}\right) \rightarrow \operatorname{Sp}\left(2(\alpha+k \infty), \mathbb{Q}_{p}\right)$ given by

$$
\iota: g \mapsto\left(\begin{array}{cc}
g & 0 \\
0 & g^{t-1}
\end{array}\right) .
$$

For any

$$
r=\left(\begin{array}{ccc}
r_{11} & \ldots & r_{12 n} \\
\vdots & \ddots & \vdots \\
r_{2 n 1} & \cdots & r_{2 n 2 n}
\end{array}\right) \in \operatorname{Sp}\left(2 k, \mathbb{Q}_{p}\right)
$$

consider the matrix $\sigma(r)=1_{2 \alpha} \oplus\left(r \otimes 1_{\infty}\right)$,

$$
\sigma(r):=\left(\begin{array}{ccccc}
1_{\alpha} & 0 & \ldots & 0 & 0 \\
0 & r_{11} \cdot 1_{\infty} & \ldots & 0 & r_{1 k} \cdot 1_{\infty} \\
\vdots & \vdots & \ddots & \vdots & \vdots \\
0 & 0 & \ldots & 1_{\alpha} & 0 \\
0 & r_{11} \cdot 1_{\infty} & \ldots & 0 & r_{1 k} \cdot 1_{\infty}
\end{array}\right)
$$

This matrix is not contained in $\operatorname{Sp}\left(2(\alpha+k \infty), \mathbb{Q}_{p}\right)$, because it is not finitary. However, the map

$$
q \mapsto \sigma\left(r^{-1}\right) q \sigma(r)
$$


is an outer automorphism of $\operatorname{Sp}\left(2(\alpha+k \infty), \mathbb{Q}_{p}\right)$. Emphasize that this automorphism fixes the subgroup $\mathbf{K}=\mathrm{O}\left(\infty, \mathbb{Z}_{p}\right)$.

We consider the representation $\rho(r)$ of $\mathrm{GL}\left(\alpha+k \infty, \mathbb{Q}_{p}\right)$ given by the formula

$$
\rho_{r}(g)=\mathrm{We}\left(\sigma\left(r^{-1}\right) \iota(g) \sigma(r)\right),
$$

where $\mathrm{We}(\cdot)$ is the Weil representation, see Subsection 3.16 .

Recall that the Weil representation is projective.

Lemma 7.1 The representation $\rho_{r}$ is equivalent to a linear representation, i.e., there is a function (a trivializer) $\gamma: \mathbf{G} \rightarrow \mathbb{C}^{\times}$such that $\gamma(g) \rho_{r}(g)$ is a linear representation.

Proof. First, the restriction of the Weil representation of $\operatorname{Sp}\left(2 n, \mathbb{Q}_{p}\right)$ to $\operatorname{GL}\left(n, \mathbb{Q}_{p}\right)$ is linear, see (3.8). Therefore, restricting the Weil representation to each finite-dimensional group $G_{j}=\mathrm{GL}\left(\alpha+k j, \mathbb{Q}_{p}\right)$ we get a representation equivalent to a linear representation (for finite-dimensional groups the automorphism (7.1) is inner). Denote by $\gamma_{j}(g)$ the trivializer for $G_{j}$. Ratio $\gamma(g)_{j} / \gamma(g)_{j+1}$ of two trivializers is a character $G_{j} \rightarrow \mathbb{C}^{\times}$. All characters of $G_{j} \rightarrow \mathbb{C}^{\times}$has the form $\varphi(\operatorname{det} h)$, where $\varphi$ is a character $\mathbb{Q}^{\times} \rightarrow \mathbb{C}^{\times}$. Correcting $\gamma_{j+1}(g) \mapsto \gamma_{j+1}(g) \psi(\operatorname{det} g)$, we can assume that $\gamma_{j+1}(g)=\gamma_{j}(g)$ on $G_{j}$.

In this way we choose a trivializer $\gamma$ on the whole group G. Restriction of $\gamma$ to $\mathrm{O}\left(\infty, \mathbb{Z}_{p}\right)$ must be a character on $\mathrm{O}\left(\infty, \mathbb{Z}_{p}\right) \rightarrow \mathbb{C}^{\times}$. The only non-trivial character is $\operatorname{det}(u)= \pm 1$. We change the trivializer $\gamma(g)$ to $\operatorname{det}(g) \gamma(g)$.

Lemma 7.2 In the model of Subsection 3.16, the subspace $L^{2}\left(\mathcal{E}_{\alpha+k \infty}\right)^{\mathbf{K}}$ of $\mathbf{K}$ fixed vectors of $\rho_{r}$ coincides with the space of functions of the form

$$
f\left(z_{1}, \ldots, z_{\alpha}\right) I\left(z_{\alpha+1}\right) I\left(z_{\alpha+2}\right) \ldots
$$

Proof. Without loss of generality, we can set $\alpha=0$. We regard $\mathcal{E}_{k \infty}$ as the space of $\infty \times k$ matrices $Z=\left\{z_{i j}\right\}$ with elements in $\mathbb{Q}_{p}$ (all but a finite number of matrix elements are in $\left.\mathbb{Z}_{p}\right)$. The group $\mathbf{K}=\mathrm{O}\left(\infty, \mathbb{Z}_{p}\right)$ acts by left multiplications

$$
\mathrm{We}(u) f(Z)=f(Z u) .
$$

We must show that $\prod_{i j} I\left(z_{i j}\right)$ is a unique $\mathrm{O}\left(\infty, \mathbb{Z}_{p}\right)$-invariant function in $L^{2}\left(\mathcal{E}_{k \infty}\right)$. Equivalently, $\mathbb{Z}_{p}^{k \infty}$ is a unique invariant subset of finite positive measure.

The group $\mathrm{O}\left(\infty, \mathbb{Z}_{p}\right)$ contains the group $S(\infty)$ of finitely supported permutations of the set $\mathbb{N}$. According zero-one law (see, e.g., 45], §4.1), the action of $S(\infty)$ on the set $\mathbb{Z}_{p}^{k \infty} \subset \mathcal{E}_{k \infty}$ is ergodic. Let $\Omega \subset \mathcal{E}_{k \infty}$ be an invariant set. Let $\xi \in \mathcal{E}_{k \infty} \backslash \mathbb{Z}_{p}^{k \infty}$. Assume that the measure of the set $\Omega \cap\left(\xi+\mathbb{Z}_{p}^{k \infty}\right)$ is non-zero, say $\nu_{0}$. Since $\Omega$ is $S(\infty)$-invariant, for any $\mathfrak{s} \in S(\infty)$, the set $\Omega \cap\left(\xi \mathfrak{s}+\mathbb{Z}_{p}^{k \infty}\right)$ has the same measure $\nu_{0}$. However there is a countable number of disjoint sets of the form $\xi \mathfrak{s}+\mathbb{Z}_{p}^{k \infty}$, therefore the measure of $\Omega$ is infinite. 
Corollary 7.3 Let $\alpha=0$. Then the representation $\rho_{r}$ contains a unique irreducible $\mathbf{K}$-spherical representation of $\mathbf{G}$.

Proof. We take the $\mathbf{G}$-cyclic span of the unique $\mathbf{K}$-fixed vector.

Next, consider the subgroup $\operatorname{GL}\left(1, \mathbb{Q}_{p}\right) \subset \operatorname{Sp}\left(2 k, \mathbb{Q}_{p}\right)$ consisting of matrices $\left(\begin{array}{cc}\lambda \cdot 1_{k} & 0 \\ 0 & \lambda^{-1} \cdot 1_{k}\end{array}\right)$, where $\lambda \in \mathbb{Q}_{p}^{\times}$.

Lemma 7.4 If $r, r^{\prime} \in \operatorname{Sp}\left(2 k, \mathbb{Q}_{p}\right)$ are contained in the same double coset

$$
\mathrm{GL}\left(1, \mathbb{Q}_{p}\right) \backslash \mathrm{Sp}\left(2 k, \mathbb{Q}_{p}\right) / \mathrm{Sp}\left(2 k, \mathbb{Z}_{p}\right),
$$

then $\rho_{r} \simeq \rho_{r^{\prime}}$.

Proof. First, if $q \in \mathrm{GL}\left(1, \mathbb{Q}_{p}\right)$, then the automorphism (7.1) fixes the subgroup GL $\left(\alpha+k \infty, \mathbb{Q}_{p}\right)$.

Second, if $t \in \operatorname{Sp}\left(2 k, \mathbb{Z}_{p}\right)$, then $\sigma(t)$ is contained in the group $\mathbf{S p}$ of automorphisms of the infinite object of the Nazarov category. Therefore the operator $\mathrm{We}(\sigma(t))$ is well-defined, it intertwines $\rho_{r}$ and $\rho_{r t}$.

7.2. Relation of characteristic functions and representations. By Lemma 7.2. we can identify the space of $\mathbf{K}$-fixed vectors of $\rho_{r}$ and the space of the Weil representation of $\operatorname{Sp}\left(2 \alpha, \mathbb{Q}_{p}\right)$.

Theorem 7.5 The representation of the semigroup $\mathbf{K} \backslash \mathbf{G} / \mathbf{K}$ in the space of $\mathbf{K}$-fixed vectors of $\rho_{r}$ is given by the formula

$$
\bar{\rho}_{r}(\mathfrak{g})=s \cdot \mathrm{We}\left(\chi_{\mathfrak{g}}\left(r \mathbb{Z}_{p}^{2 k}, r \mathbb{Z}_{p}^{2 k}\right)\right), \quad s \in \mathbb{C}^{\times} .
$$

Proof. We use the notation and statements of Subsection 3.16 Let $\mathcal{V}$ and $\mathcal{H}$ be the same as in Section 4. Let $Y=\mathcal{V}_{2 k \infty}, W=\mathcal{V} \oplus Y$. The operator of projection $\mathcal{H}(\mathcal{V} \oplus Y)$ to $\mathcal{H}(V \oplus Y)^{\mathbf{K}} \simeq \mathcal{H}(V)$ is $\mathrm{We}\left(\theta_{W}^{V}\right)$. Therefore

$$
\bar{\rho}(\mathfrak{g})=s^{\prime} \cdot \operatorname{We}\left(\theta_{W}^{V}\right) \mathrm{We}\left(\sigma\left(r^{-1}\right) \iota(g) \sigma(r)\right) \mathrm{We}\left(\theta_{W}^{V}\right)
$$

as an operator $L^{2}\left(\mathcal{E}_{\alpha+k \infty}\right)^{\mathbf{K}} \rightarrow L^{2}\left(\mathcal{E}_{\alpha+k \infty}\right)^{\mathbf{K}}$. The operator

$$
\mathrm{We}\left(\lambda_{W}^{V}\right): L^{2}\left(\mathbb{Q}_{p}^{\alpha}\right) \rightarrow L^{2}\left(\mathcal{E}_{\alpha+k \infty}\right)
$$

is an operator of isometric embedding, the image is $\mathcal{H}\left(V \oplus V_{2 k \infty}\right)^{\mathbf{K}}$. Therefore we can write $\bar{\rho}(\mathfrak{g})$ as

$$
\begin{array}{r}
\bar{\rho}(\mathfrak{g})=s^{\prime \prime} \cdot \operatorname{We}\left(\lambda_{W}^{V}\right)^{*} \operatorname{We}\left(\theta_{W}^{V}\right) \operatorname{We}\left(\sigma\left(r^{-1}\right) \iota(g) \sigma(r)\right) \operatorname{We}\left(\theta_{W}^{V}\right) \operatorname{We}\left(\lambda_{W}^{V}\right)= \\
=s^{\prime \prime \prime} \cdot \operatorname{We}\left(\lambda_{W}^{V}\right)^{*} \operatorname{We}\left(\sigma\left(r^{-1}\right) \iota(g) \sigma(r)\right) \operatorname{We}\left(\lambda_{W}^{V}\right)= \\
=s^{\prime \prime \prime \prime} \cdot \operatorname{We}\left[\left(\lambda_{W}^{V}\right)^{*} \sigma\left(r^{-1}\right) \iota(g) \sigma(r) \lambda_{W}^{V}\right] .
\end{array}
$$

Next, $\sigma(r) \lambda_{W}^{V}: V \rightrightarrows V \oplus Y$ is a direct sum of $1_{V} \subset V \oplus V$ and the lattice in $Y$ given by

$$
\left.\sigma(r) Y(\mathbb{O})=\sigma(r)\left(H(\mathbb{O}) \otimes \mathbb{O}^{\infty}\right)=(r H(\mathbb{O})) \otimes \mathbb{O}^{\infty}\right) .
$$


We apply Subsection 5.12 for the expression in square brackets in (7.2).

7.3. A more general construction. Consider the embedding

$$
\iota_{l}: \mathrm{GL}\left(\alpha+k \infty, \mathbb{Q}_{p}\right) \rightarrow \operatorname{Sp}\left(2 l \alpha+2 l k \infty, \mathbb{Q}_{p}\right)
$$

given by

$$
g \mapsto\left(\begin{array}{cccccc}
g & \ldots & 0 & 0 & \ldots & 0 \\
\vdots & \ddots & \vdots & \vdots & \ddots & \vdots \\
0 & \ldots & g & 0 & \ldots & 0 \\
0 & \ldots & 0 & g^{t-1} & \ldots & 0 \\
\vdots & \ddots & \vdots & \vdots & \ddots & \vdots \\
0 & \ldots & 0 & 0 & \ldots & g^{t-1}
\end{array}\right) .
$$

This is a $2 l \times 2 l$ block matrix, each block of this matrix has size $(\alpha+k \infty) \times$ $(\alpha+k \infty)$.

Next, for a matrix $r \in \operatorname{Sp}\left(2 k l, \mathbb{Q}_{p}\right)$ we take

$$
\sigma(r):=1_{2 \alpha l} \oplus\left(r \otimes 1_{\infty}\right)
$$

and consider the representation of $\operatorname{GL}\left(\alpha+k \infty, \mathbb{Q}_{p}\right)$ given by

$$
\rho_{r}(g)=\mathrm{We}\left(\sigma(r)^{-1} \iota_{l}(g) \sigma(r)\right) .
$$

Set $\alpha=0$. As above, each representation $\rho_{r}$ of $\mathbf{G}=\mathrm{GL}\left(k \infty, \mathbb{Q}_{p}\right)$ contains a unique $\mathbf{K}$-spherical subrepresentation.

Conjecture 7.6 Any $\mathbf{K}$-spherical representation of $\mathrm{GL}\left(k \infty, \mathbb{Q}_{p}\right)$ is a subrepresentation in $\varphi(\operatorname{det}(g)) \rho_{r}(g)$, where $\varphi=\varphi_{r}: \mathbb{Q}_{p}^{\times} \rightarrow \mathbb{C}^{\times}$is a character. Representations $\rho_{r}$ are parametrized by the set

$$
\bigcup_{l} \mathrm{GL}\left(l, \mathbb{Q}_{p}\right) \backslash \operatorname{Sp}\left(2 k l, \mathbb{Q}_{p}\right) / \operatorname{Sp}\left(2 k l, \mathbb{Z}_{p}\right) .
$$

\section{References}

[1] Wildberger, N.J. Hypergroups, symmetric spaces, and wrapping maps. in Heyer, Herbert (ed.), Probability measures on groups and related structures. $X I$. Proceedings of the meeting held in Oberwolfach, Germany, October 2329, 1994. World Scientific 406-425 (1995).

[2] Gelfand, I. M. Spherical functions in symmetric Riemann spaces. (Russian) Doklady Akad. Nauk SSSR (N.S.) 70, (1950), 5-8.

[3] Godement, R. A theory of spherical functions. I. Trans. Am. Math. Soc. $73,496-556(1952)$

[4] Iwahori, N. On the structure of a Hecke ring of a Chevalley group over a finite field. J. Fac. Sci., Univ. Tokyo, Sect. I 10, 215-236 (1964). 
[5] Iwahori, N.; Matsumoto, H. On some Bruhat decomposition and the structure of the Hecke rings of p-adic Chevalley groups. Publ. Math., Inst. Hautes Etud. Sci. 25, 5-48 (1965).

[6] Koornwinder, T. H. Jacobi functions and analysis on noncompact semisimple Lie groups. In Special functions: group theoretical aspects and applications, 1-85, Math. Appl., Reidel, Dordrecht, 1984.

[7] Olshanski, G.I. Unitary representations of infinite dimensional pairs $(G, K)$ and the formalism of $R$. Howe. In Representation of Lie groups and related topics, Adv. Stud. Contemp. Math. 7, 269-463 (1990).

[8] Neretin, Yu. A. Categories of symmetries and infinite-dimensional groups. Oxford University Press, New York, 1996.

[9] Neretin, Yu. A. Lectures on Gaussian integral operators and classical groups. European Mathematical Society (EMS), Zürich, 2011.

[10] Brodski, M. S., Unitary operator colligations and their characteristic functions. Russ. Math. Surveys, 1978, 33:4, 159-191.

[11] Olshanski, G. I. On semigroups related to infinite-dimensional groups. In: Topics in representation theory (A. A. Kirillov, ed.). Advances in Soviet Math., vol. 2. Amer. Math. Soc., Providence, R.I., 1991, 67-101.

[12] Neretin, Yu.A. On degeneration of convolutions of double cosets at infinitedimensional limit. Preprint, http://arxiv.org/abs/1211.6149

[13] Ismagilov, R.S., Elementary spherical functions on the groups $\operatorname{SL}(2, P)$ over a field $P$, which is not locally compact with respect to the subgroup of matrices with integral elements. Math. USSR-Izvestiya, 1967, 1:2, 349-380.

[14] Ismagilov, R.S., Spherical functions over a normed field whose residue field is infinite. Funct. Anal. and Its Appl. Volume 4, N. 1, 37-45.

[15] Olshanski, G. I. New "large" groups of type I. Current problems in mathematics, Vol. 16 (Russian), pp. 31-52, 228, Akad. Nauk SSSR, Vsesoyuz. Inst. Nauchn. i Tekhn. Informatsii (VINITI), Moscow, 1980. English transl. J. Sov. Math. 18 (1982) 22-3

[16] Neretin, Yu. A. Sphericity and multiplication of double cosets for infinitedimensional classical groups. Funct. Anal. Appl., 45:3 (2011), 225-239.

[17] Neretin, Yu. A. Infinite symmetric group and combinatorial descriptions of semigroups of double cosets. Preprint, arXiv:1106.1161

[18] Neretin, Yu. A., Infinite tri-symmetric group, multiplication of double cosets, and checker topological field theories. Int. Math. Res. Notes, 2012 (2011), 3, 501-523. 
[19] Neretin, Yu. A. Multiplication of conjugacy classes, colligations, and characteristic functions of matrix argument. Preprint, arXiv:1211.7091

[20] Neretin, Yu. A. Multi-operator colligations and multivariate spherical functions. Anal. and Math. Physics, 1 (2011) 2-3, 121-138.

[21] Garnett, J. B. Bounded analytic functions. Academic Press, Inc. New YorkLondon, 1981

[22] Livshits, M. S. On a certain class of linear operators in Hilbert space. Mat. Sb., N. Ser. 19(61), 239-262 (1946); English transl. in Amer. Math. Soc. Transl. (Ser. 2), Vol. 13, 61-83 (1960)

[23] Potapov, V. P. The multiplicative structure of J-contractive matrix functions. Trudy Moskov. Mat. Obshchestva. 4 (1955), 125-236; English transl. Amer. Math. Soc. Transl. (2) 15 (1960) 131-243

[24] Olshanski, G. I. Infinite-dimensional classical groups of finite R-rank: description of representations and asymptotic theory. Funct. Anal. and Appl., 1984, 18:1, 22-34

[25] Olshanski, G. I. Unitary representations of infinite-dimensional pairs $(G, K)$ and the formalism of $R$. Howe. Soviet Math. Dokl. 27 (1982), no. 2, 290-294.

[26] Olshanski, G.I. The problem of harmonic analysis on the infinitedimensional unitary group. J. Funct. Anal., 205 (2003), 464-524.

[27] Borodin, A., Olshanski, G. Harmonic analysis on the infinite-dimensional unitary group and determinantal point processes. Ann. of Math. (2) 161 (2005), no. 3, 1319-1422.

[28] Olshanski, G.I., Unitary representations of $(G, K)$-pairs connected with the infinite symmetric group $S(\infty)$. Leningr. Math. J. 1, No.4, 983-1014 (1990).

[29] Kerov, S., Olshanski, G., Vershik, A. Harmonic analysis on the infinite symmetric group. Invent. Math. 158 (2004), No. 3, 551-642.

[30] Vershik,A. M.; Kerov, S. V. Four drafts on the representation theory of the group of infinite matrices over a finite field. J. of Math. Sci. (New York), 2007, 147:6, 7129-7144

[31] Neretin, Yu.A. The space $L^{2}$ on semi-infinite Grassmannian over finite field, Adv. Math., 250 (2014), 320-350

[32] Gorin, V., Kerov S., Vershik A. Finite traces and representations of the group of infinite matrices over a finite field. Preprint, arXiv:1209.4945

[33] Nazarov, M. Oscillator semigroup over a non-Archimedean field. J. Funct. Anal. 128 (1995), no. 2, 384-438. 
[34] Nazarov, M.; Neretin, Yu.; Olshanskij, G. Semi-groupes engendrés par la représentation de Weil du groupe symplectique de dimension infinie. C. R. Acad. Sci. Paris Sér. I Math. 309 (1989), no. 7, 443-446.

[35] Neretin, Yu. A. Hua measures on the space of p-adic matrices and inverse limits of Grassmannians. Izvest. Math., 77, 5 (2013), 941-953.

[36] Neretin, Yu. A. Hua-type integrals over unitary groups and over projective limits of unitary groups. Duke Math. J. 114, No.2, 239-266 (2002).

[37] Neretin, Yu. A. Combinatorial analogues of the group of diffeomorphisms of the circle. Russian Acad. Sci. Izv. Math. 41 (1993), no. 2, 337-349

[38] Neretin, Yu.A. On p-adic colligations and 'rational maps' of Bruhat-Tits trees, Preprint, arXiv:1301.5453

[39] Baker, M. An introduction to Berkovich analytic spaces and nonArchimedean potential theory on curves. in Savitt, D. (ed.) et al.,p-adic geometry. Providence, RI: American Mathematical Society, 123-174 (2008).

[40] Conrad, B. Several approaches to non-archimedean geometry. Savitt, D. (ed.) et al., p-adic geometry. Providence, RI: American Mathematical Society, 9-63 (2008).

[41] Weil, A. Basic number theory. Springer-Verlag, New York, 1967.

[42] Garrett, P. Buildings and classical groups. Chapman \& Hall, London, 1997.

[43] Neretin, Yu.A. On compression of Bruhat-Tits buildings. J. Math. Sci. (N. Y.), 2006, 138:3, 5722-5726.

[44] Hatcher, A. Algebraic topology. Cambridge University Press, (2002);

[45] Shiryaev, A. N. Probability, Springer, 1984

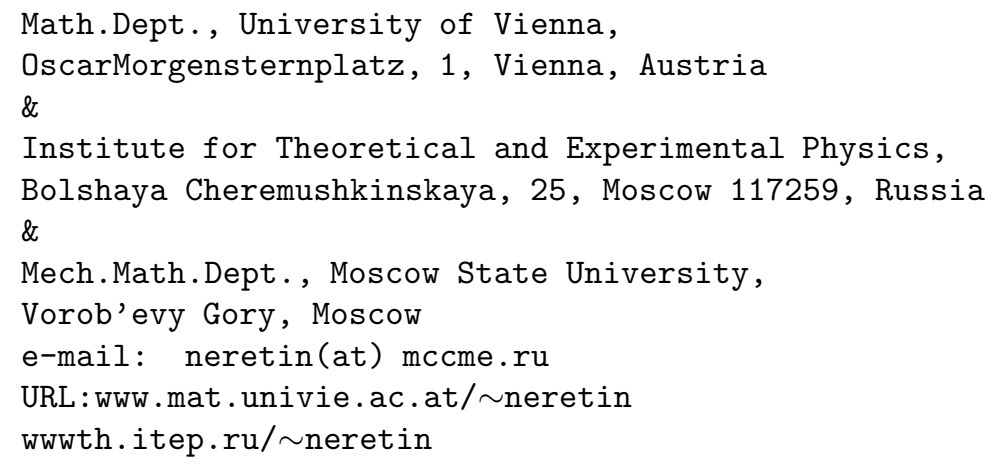

